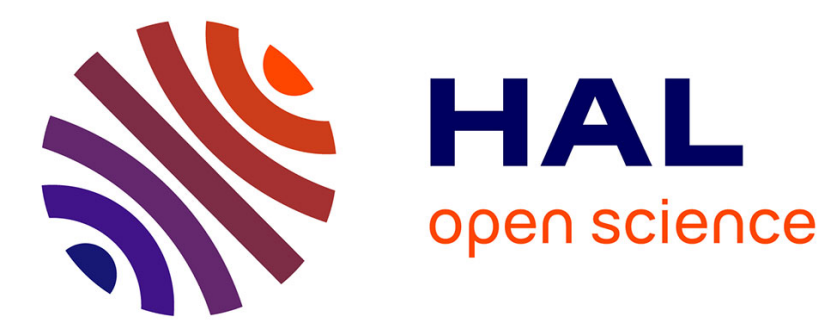

\title{
Current-driven transverse domain wall oscillations in perpendicular spin-valve structures
}

M V Lubarda, M Kuteifan, C.-H Lambert, V. Lomakin, E. E. Fullerton, S. Mangin

\section{- To cite this version:}

M V Lubarda, M Kuteifan, C.-H Lambert, V. Lomakin, E. E. Fullerton, et al.. Current-driven transverse domain wall oscillations in perpendicular spin-valve structures. Physical Review B: Condensed Matter (1978-1997), 2020, 102, pp.014405. 10.1103/PhysRevB.102.014405 . hal-02893574

\section{HAL Id: hal-02893574 \\ https://hal.univ-lorraine.fr/hal-02893574}

Submitted on 8 Jul 2020

HAL is a multi-disciplinary open access archive for the deposit and dissemination of scientific research documents, whether they are published or not. The documents may come from teaching and research institutions in France or abroad, or from public or private research centers.
L'archive ouverte pluridisciplinaire $\mathbf{H A L}$, est destinée au dépôt et à la diffusion de documents scientifiques de niveau recherche, publiés ou non, émanant des établissements d'enseignement et de recherche français ou étrangers, des laboratoires publics ou privés. 


\title{
Current driven transverse domain wall oscillations in perpendicular spin valve structure
}

\author{
M. V. Lubarda ${ }^{1-4}$, M. Kuteifan ${ }^{2,3}$, C.-H. Lambert ${ }^{2}$, V. Lomakin ${ }^{3}$, E. E. Fullerton ${ }^{3}$, S. Mangin ${ }^{2}$ \\ ${ }^{1}$ Faculty of Polytechnics, University of Donja Gorica, Oktoih 1, 81000 Podgorica, Montenegro \\ ${ }^{2}$ Université de Lorraine, CNRS, Institut Jean Lamour, F-54000 Nancy, France \\ ${ }^{3}$ Center for Memory and Recording Research, University of California, San Diego, California 92093-0401, USA \\ ${ }^{4}$ Department of Mechanical and Aerospace Engineering, University of California, San Diego, California 92093 -
}

0411, USA

\begin{abstract}
Spin-transfer-driven oscillations of a transverse domain wall confined to a perpendicular spin-valve structure are investigated using a one-dimensional model. The stack consists of a polarizer, nonmagnetic spacer, soft free layer, and pinned magnetic layer. It is found that the domain-wall oscillation frequency is a non-monotonic, highly asymmetrical function of applied electric current, showing a strong dependence on the current direction and the relative strengths of the interfacial and bulk spin-transfer torques. Micromagnetic analysis reveals that the surprising and atypical oscillator response is due to an interplay between the interfacial spin-transfer torque, the bulk spintransfer torque, the exchange torque, and the damping torque. The underlying physical and materials responses are examined, including the important role of the domain-wall twist. The competitions between the involved torques under different operating conditions suggest that the oscillator could serve as a model system to investigate magnetic and spintronic phenomena at the nanoscale. The observed current-dependent twisting of the free-layer magnetization about the axis of precession may further be found interesting for investigations of the interaction between spin-polarized current and chiral spin structures.
\end{abstract}

\section{INTRODUCTION}

Spin torque nano-oscillators (STNOs) are nanoscale microwave generators holding great technological promise for telecommunications, microwave-assisted magnetic recording (MAMR), neuromorphic computing $^{1,2}$ and other high-frequency applications ${ }^{3-6}$. In STNOs, an electric current, typically DC, drives the magnetization into precession by means of spin-transfer torque (STT) ${ }^{7-11}$ or spin-orbit torque (SOT) $)^{12-15}$. The magnetization precession is commonly detected through the giant magnetoresistance (GMR) ${ }^{16,17}$ or tunneling magnetoresistance (TMR) ${ }^{18}$ effect as oscillations characteristically in the high $\mathrm{MHz}$ to low $\mathrm{THz}$ frequency range. STNOs have lately attracted considerable research interest due to their technological appeal, including nanoscale dimensions, integrability with CMOS technology, high operating frequency, non-linear response, and frequency tunability ${ }^{19-32}$.

Most widely investigated STNOs are point-contact geometries ${ }^{22,33-37}$ and pillar-structured STNOs based on magnetoresistive spin valves ${ }^{38-41}$ and magnetic tunnel junctions (MTJs) ${ }^{23,42-46}$. Nanowire-based STNOs have been considered, as well ${ }^{27,47,48}$. Depending on the specifics of the system, the magnetic object undergoing oscillations can be a magnetic domain ${ }^{23,39-46}$, domain wall $(\mathrm{DW})^{47,49-57}$, vortex ${ }^{38,58-60}$, or skyrmion $^{30,61-65}$. Within each STNO category, numerous device designs have been proposed with the aim of achieving improved performance characteristics, including higher operating frequency, narrower linewidth, greater power output, and enhanced tunability. Many designs have demonstrated their usefulness as model systems for fundamental investigations of physical processes occurring in magnetic and spintronic systems at the nanoscale. Particularly, STNOs have been used for the study of angular momentum transfer between spin-polarized current and local magnetization, topological excitations, vortex dynamics, mode hopping, spin wave generation, and phase locking. 
In this work, we consider from a theoretical and numerical standpoint the operation and properties of allperpendicular spin-valve STNOs where a DW is formed in the free layer under the application of an electric current. . The system is comprised of a multilayer stack consisting of a polarizer, nonmagnetic spacer layer, magnetically soft free layer, and pinned magnetic layer (Fig. 1a). The polarizer and pinned magnetic layers are assumed to have strong perpendicular magnetic anisotropy and considered fixed in these discussions. An electric current applied perpendicularly through the stack drives the oscillator dynamics in the free layer. An alternative three-terminal device involving a nonmagnetic heavy-metal layer at the base of the free magnetic layer is also discussed.

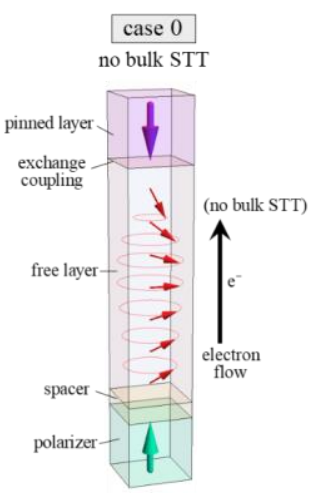

(a)

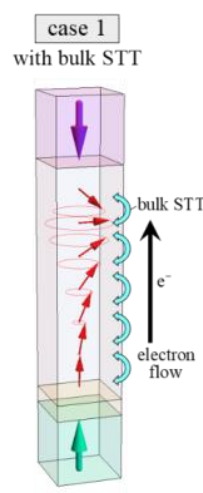

(b)

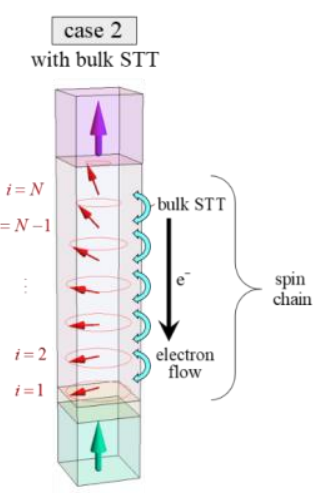

(c)

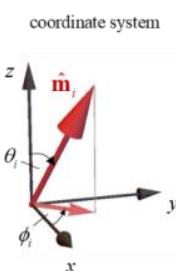

(d)

Figure 1: Schematic illustration of an all-perpendicular spin valve in the three cases considered: (a) case 0 , (b) case 1 , (c) case 2 . In cases 0 and 1, the polarizer and pinned layer magnetizations are oppositely oriented and electron flow is in the direction from polarizer to pinned layer. In case 2 , the magnetizations of pinned layer and polarizer are equally oriented, and the electron flow is in the reverse direction. In case 0 , the electron flow within the bulk of the free layer is not spin polarized, while in cases 1 and 2 it is. The free layer magnetization is modeled as a chain of spins, with the adopted spin-numbering scheme shown in Fig. 1c. Figure 1d shows the coordinate system with

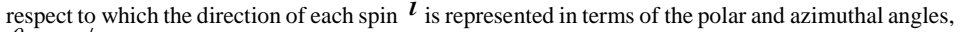
$\theta_{i}$ and $\phi_{i}$, respectively.

In the sections to follow, we first demonstrate the operation of the STNO in three regimes. Then, we present a reduced-complexity computational model and describe the observed dependence of the oscillation frequency on the applied electric current, the structural and material properties of the free layer, and the spin polarization factor for different operating configurations of the STNO. We finally examine and discuss the underlying physics responsible for the unique dynamical response of DW-based spin-valve STNOs, including reference to the nature of the competition between the participating interactions and the role of DW twist. Further details are also given in the Supplementary Material.

\section{STRUCTURAL CONFIGURATION AND OPERATION}

We consider three cases of operation illustrated in Figs. 1a-c. Depending on the directions of applied current and pinned layer magnetization, and whether bulk STT is negligible (case 0; Fig. 1a) or appreciable (cases 
1 and 2; Figs. 1b, c), strikingly different frequency responses are obtained (Figs. Erreur ! Source du renvoi introuvable.a-c), with particularly surprising and atypical frequency-current characteristics observed in cases 0 (Fig. 2a) and 2 (Fig. 2e). Frequency results in Fig. 2 were obtained by simulating the magnetization dynamics of the free layer based on the spin-chain model outlined in section III (calculation details can be found in the Appendix).

(a)

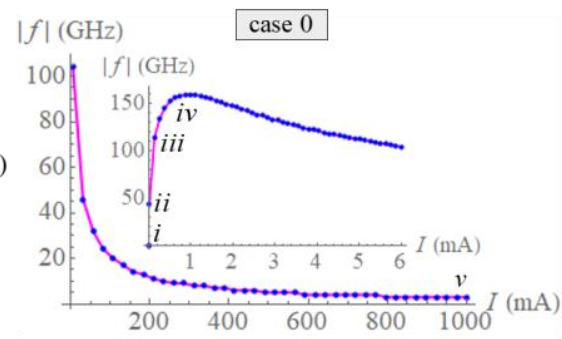

(b)

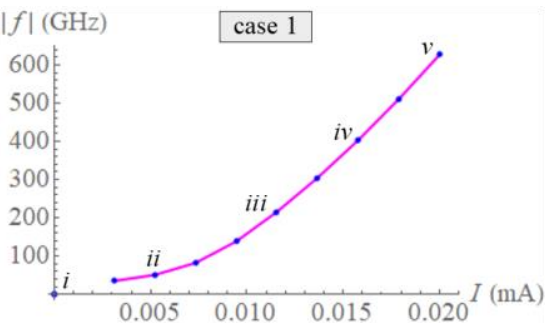

(c)

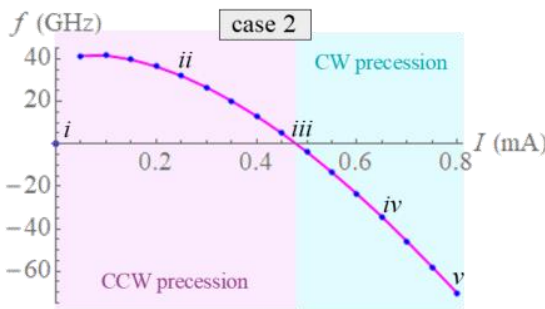

(d)

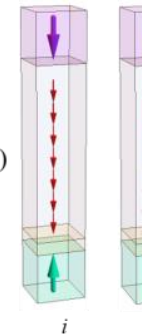

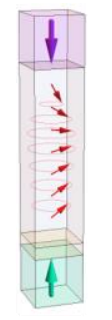

iii
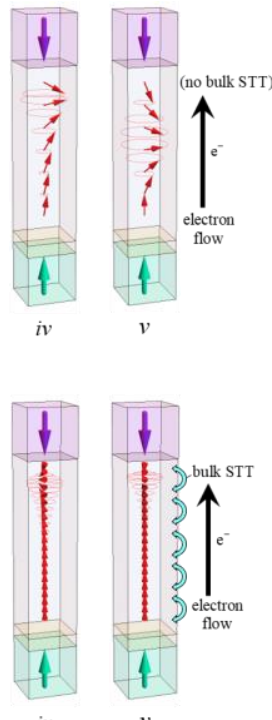

$v$

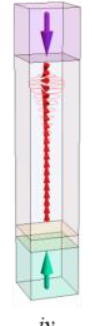

(e)

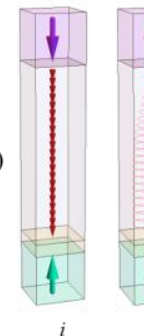

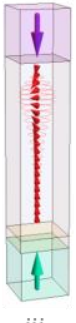
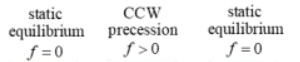

$\underset{\substack{\text { precession } \\ f<0}}{\mathrm{CW}}$

precession
$f<0$

(f)
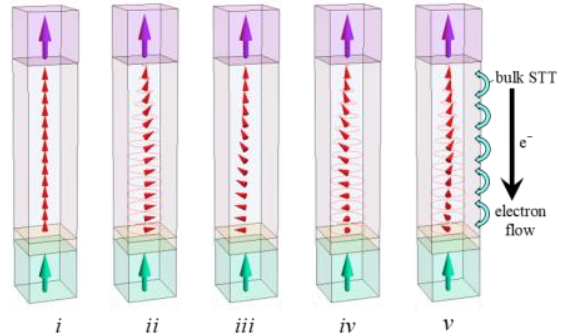

Figure 2: (a-c) Frequency as a function of applied current for cases 0, 1, and 2 (Fig. 1a-c). (d-f) Corresponding spin configurations during steady-state precession about the vertical axis for several illustrative current values. (Spin valve not drawn to scale for convenience of illustration. Geometrical and material parameters are given in the main text. Conversion from current amplitude to current density for all cases: $0.025 \mathrm{~mA} \leftrightarrow 10^{8} \mathrm{~A} / \mathrm{cm}^{2}$.)

In all cases considered (Figs. 1a-c), upon application of electric current, the spins of the itinerant electrons flowing through the spacer layer interact with the local magnetic moment of the polarizer and free layers, causing a transfer of angular momentum between them. This is known as the STT effect ${ }^{7,8,11}$. Because it is local in nature, occurring primarily at the interfaces of the magnetic and spacer layers, it is referred to as 
the interfacial STT effect. As discussed later, the bulk STT effect ${ }^{9}, 10$ also has an important role in the STNO operation.

For electron flow from the polarizer toward the free layer (cases 0 and 1; Figs. 1a and b, respectively), the resulting interfacial STT tends to orient the magnetization of the free layer in the direction of the polarizer magnetization. Conversely, for electron flow in the opposite direction (case 2, Fig. 1c), the interfacial STT tends to orient the magnetization of the free layer in the direction opposite to that of the polarizer magnetization. In all cases, the tendency is for a DW to be introduced into the free layer from the end adjacent to the polarizer.

In case 0 (Fig. 1a), as the current is initially increased, the magnetization of the free layer cants due to the action of the interfacial STT, and precession begins about the pinned-layer interlayer exchange field with a frequency that is positively correlated with current (inset of Fig. Erreur! Source du renvoi introuvable.a). For sufficiently large currents, a DW is introduced into the free layer (Fig. Erreur ! Source du renvoi introuvable.b-iii) and moves up toward the pinned layer as current is further increased. Remarkably, a maximum precessional frequency is eventually reached (Fig. Erreur! Source du renvoi introuvable.a-iv), at which time the DW is closest to the pinned layer (Fig. Erreur ! Source du renvoi introuvable.b-iv). Further increases in current results in a reduction of precessional frequency and a downward movement of the DW away from the pinned layer. The frequency asymptotically approaches zero (Fig. Erreur ! Source du renvoi introuvable.a) and the DW position converges to the midpoint of the free layer (Fig. Erreur ! Source du renvoi introuvable.b-v) as $I \rightarrow \infty$.

In cases 1 and 2 (Figs. $1 \mathrm{~b}$ and c), when the spin polarization of the electric current within the bulk of the free layer is non-negligible there is an additional bulk STT exerted within the free magnetic layer due to the interaction between the spin-polarized current and the spatially varying local magnetic moment ${ }^{9}$. The primary action of the bulk STT is to push the DW in the direction of the electron flow ${ }^{10}$.

In case 1 (Fig. 1b), the bulk STT drives the DW toward the pinned layer, against which it is progressively compressed as current is increased. Since the interlayer exchange torque, which generates the precession, is larger when the DW is more compressed against the pinned layer, and because the in-plane component of the free-layer net magnetic moment (precessional inertia) is smaller with greater DW confinement (Fig. Erreur ! Source du renvoi introuvable.e), more rapid oscillations are observed at larger current (Fig. Erreur ! Source du renvoi introuvable.b).

Conversely, in case 2 (Fig. 1c), the bulk STT pushes the DW in the reverse direction, toward the polarizer. For relatively low currents, precession is driven by the interlayer exchange torque supplied by the pinned layer (top), hence precession in the $\mathrm{CCW}$ direction $(\underline{f>0})$, as can be inferred from the pinned-layer magnetization orientation. As the current is raised, the frequency of precession reduces, reaching zero (static equilibrium) for a certain current value (Fig. Erreur ! Source du renvoi introuvable.c). With a further increase in current, precession begins again, but now in the opposite, CW direction $(f<0)$, with $|f|$ positively correlated with $I$.

In all cases, under constant current, the system converges to a solution characterized by a steady-state precession of the DW magnetization about the vertical axis at some given frequency. The frequency-current relationships observed for the three cases (Fig. Erreur ! Source du renvoi introuvable.) can be understood in terms of the competition between the participating torques (Figs. 3, 4, and 5) and the twist of the spin chain that develops in case 0 and case 2, but not in case 1 (Fig. 6). The interplay between interfacial STT, bulk STT, interlayer exchange, and magnetocrystalline anisotropy, discussed here in the context of STNOs, 
may be additionally relevant to STT-MRAM designs employing elongated storage layers, which have recently been proposed as a route to enhancing lateral scalability of memory cells to sub-10nm scale ${ }^{66-68}$. Similar interactions are also expected to occur in analogous three-terminal systems involving a current path running horizontally through a non-magnetic heavy-metal layer connecting to the free layer in place of the polarizer-spacer duo ${ }^{55,69-75}$. In such devices, the role of the interfacial STT would be replaced by that of the SOT deriving from the spin-Hall effect or broken inversion symmetry at the corresponding interface ${ }^{12-15}$.

\section{ONE-DIMENSIONAL SPIN-CHAIN MODEL}

A one-dimensional (1D) model is first employed to represent the STNO in simplified form and to gain a general understanding of the underlying physics controlling STNO response highlighted in Fig. 2. Within this model, the magnetizations of the polarizer and the pinned layer are fixed. All degrees of freedom of the magnetic system are thus solely associated with the magnetization of the free layer, which we model as a chain of spins (Fig. 1c). The dynamics of the chain of spins describing the free layer magnetization is governed by the Landau-Lifshitz-Gilbert equation, consisting of the precessional- and damping-torque terms, and extended by the interfacial current-induced torque term, either interfacial STT or SOT, and the adiabatic and non-adiabatic bulk-STT terms ${ }^{14}$ :

$$
\frac{d \hat{\mathbf{m}}_{i}}{d t}=-\gamma \hat{\mathbf{m}}_{i} \times \mathbf{H}_{\mathrm{eff}, i}+\alpha \hat{\mathbf{m}}_{i} \times \frac{d \hat{\mathbf{m}}_{i}}{d t}+\boldsymbol{\tau}_{\text {interface }, i}+\boldsymbol{\tau}_{\mathrm{bSTT}, i} .
$$

In the equation above, $\hat{\mathbf{m}}_{i}$ denotes the unit direction vector of the $i$ th spin in the chain, ${ }^{t}$ (s) is the time, $\gamma\left(\mathrm{rad} \cdot \mathrm{s}^{-1} \cdot \mathrm{Oe}^{-1}\right)$ is the gyromagnetic ratio (taken as positive), and $\alpha$ (unitless)

constant of the free layer. The effective magnetic field exerted on spin $i$ is denoted by $\mathbf{H}_{\text {eff }, i}(\mathrm{Oe})$. It is equal to the sum of the applied magnetic field $\mathbf{H}_{\text {app }, i}$, the bulk exchange field ${ }_{\text {ex }, i}$, the interlayer exchange field $\mathbf{H}_{\text {iex, } i}$, and the magnetocrystalline anisotropy field $\mathbf{H}_{\text {anis }, i}$, i.e.,

$$
\mathbf{H}_{\mathrm{eff}, i}=\mathbf{H}_{\mathrm{app}, i}+\mathbf{H}_{\mathrm{ex}, i}+\mathbf{H}_{\mathrm{iex}, i}+\mathbf{H}_{\mathrm{anis}, i} .
$$

Here, we have excluded from considerations the Oersted field, estimating that its effect on magnetization dynamics is negligible considering the nanoscale size of the spin-valve structure that we model (see next section for spin-valve dimensions). We have also dropped the magnetostatic field in Eq. (1), to simplify the analysis of the interplay between interactions responsible for the observed STNO frequency-current characteristics (Figs. Erreur ! Source du renvoi introuvable.a-c), which are not qualitatively affected by dipolar coupling. Expressions for the fields in Eq. (2) are provided in the Supplementary Material.

The interfacial current-induced torque in Eq. (1), as the interlayer exchange torque, is exerted only at an end spin, specifically the bottommost spin $(i=1)$, and is modeled as

$$
\boldsymbol{\tau}_{\text {interface }, 1}=\tau_{\mathrm{FL}} \hat{\mathbf{m}}_{1} \times \hat{\mathbf{p}}+\tau_{\mathrm{DL}} \hat{\mathbf{m}}_{1} \times \hat{\mathbf{m}}_{1} \times \hat{\mathbf{p}}
$$

The direction of the unit vector $\hat{\mathbf{p}}$ and the values of the prefactors $\tau_{\mathrm{FL}}$ and $\tau_{\mathrm{DL}}$ are determined by the specifics of the mechanism responsible for the torque, device geometry, materials system, and magnetization configuration ${ }^{14}$. In the case of interfacial STT, the unit vector $\hat{\mathbf{p}}$ has the direction of the polarizer magnetization, i.e., $\hat{\mathbf{p}}=\left(\begin{array}{lll}0 & 0 & p_{z}\end{array}\right)$ with $p_{z}= \pm 1$. In case of SOT, $\hat{\mathbf{p}}$ is in the plane of the nonmagnetic heavy-metal layer and orthogonal to the direction of current flow. For details, the reader is 
referred to the Supplementary Material and elsewhere ${ }^{14}$. In our analysis, we explicitly consider the case of interfacial STT, i.e., $\boldsymbol{\tau}_{\text {interface, } 1} \equiv \boldsymbol{\tau}_{\text {iSTT,1 }}$, and shall refer to it from this stage forward by this latter symbol.

The last term $\boldsymbol{\tau}_{\mathrm{bSTT}, i}$ in the dynamical Eq. (1) represents the bulk STT, which, unlike the interfacial STT, is applied to all the spins of the spin chain . The bulk STT efficiency is considered zero in case 0 and nonnegligible in cases 1 (Fig. 1b) and 2 (Fig. 1c). While the bulk STT generally consists of an adiabatic and non-adiabatic contribution, we neglect the latter contribution in the ensuing analysis, as its effect on the results was found to not be significant. In other systems, however, the non-adiabatic contribution can play a critical role ${ }^{9,10,76-78}$. The explicit expression for the bulk STT is provided in the Supplementary Material.

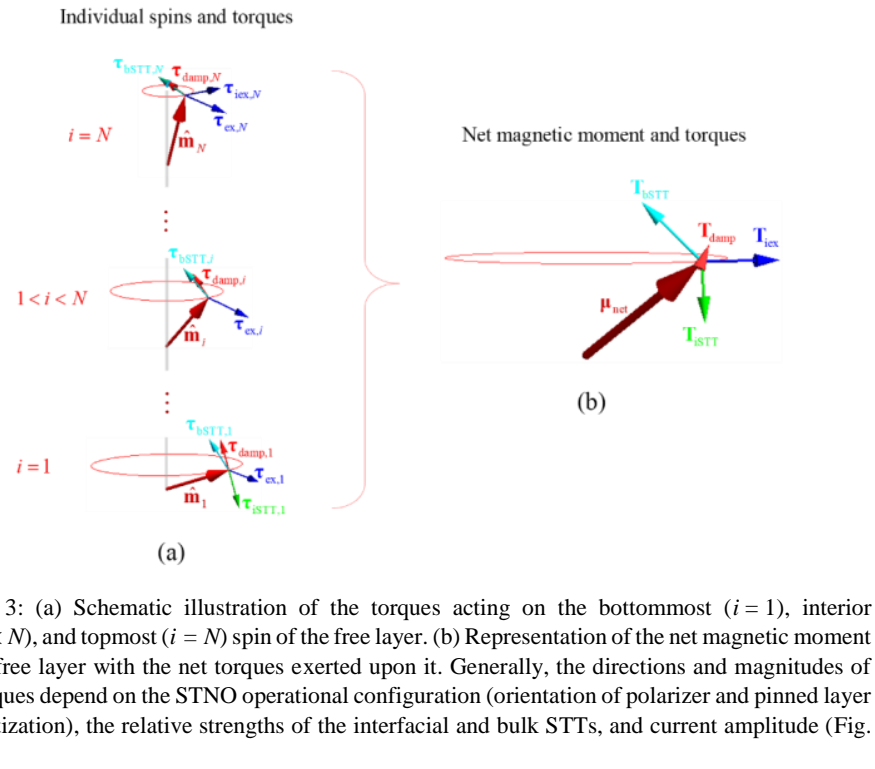

According to the first term in Eq. (1), the precessional torques due to the micromagnetic fields can be individually computed using $\boldsymbol{\tau}=-\gamma \hat{\mathbf{m}} \times \mathbf{H}$. These torques, together with the interfacial and bulk STTs, are depicted in Fig. 3a. The net magnetic moment $\boldsymbol{\mu}_{\text {net }}(\mathrm{emu})$ of the free layer and the net individual torques acting on it, $\mathbf{T}_{\text {iSTT }}, \mathbf{T}_{\text {bSTT }}, \mathbf{T}_{\text {iex }}, \mathbf{T}_{\text {damp }}(\mathrm{emu} / \mathrm{s})$, are schematically represented in Fig. 3b. Their computation is detailed in the Supplementary Material.

Several qualities of the involved torques should be pointed out, which can be deduced from their expressions given in the Supplementary Material. For a vertically magnetized polarizer, assuming that $\tau_{\mathrm{FL}}=0$ in Eq. (3) (reasonable assumption for all-metallic spin-valve devices), the interfacial STT has only a polar component, i.e., $\boldsymbol{\tau}_{\text {iSTT, } 1}=\tau_{\text {iSTT,1 }} \hat{\boldsymbol{\theta}}_{1}$, where $\hat{\boldsymbol{\theta}}_{1}=\left(\begin{array}{lll}\cos \theta_{1} \cos \phi_{1} & \cos \theta_{1} \sin \phi_{1} & -\sin \theta_{1}\end{array}\right)$ is the unit 
vector in the polar direction with respect to the first spin. Similarly, for a vertically magnetized pinned layer, the interlayer exchange torque acts only along the azimuthal direction with respect to the topmost spin, i.e., $\boldsymbol{\tau}_{\text {iex }, N}=\tau_{\text {iex }, N} \phi_{N}$, where $\boldsymbol{\phi}_{N}=\left(\begin{array}{lll}-\sin \phi_{N} & \cos \phi_{N} & 0\end{array}\right)$. In case of a planar spin structure, the bulk exchange torque and the bulk STT have the form $\boldsymbol{\tau}_{\mathrm{ex}, i}=\tau_{\mathrm{ex}, i} \hat{\boldsymbol{\phi}}_{i}$ and $\boldsymbol{\tau}_{\mathrm{bSTT}, i}=\tau_{\mathrm{bSTT}, i} \hat{\boldsymbol{\theta}}_{i}$, whereas in the presence of DW twist, i.e., for a non-planar spin structure, these torques have finite projections in both the polar and azimuthal directions, i.e., $\boldsymbol{\tau}_{\mathrm{ex}, i}=\tau_{\mathrm{ex}, i}^{\Theta} \hat{\boldsymbol{\theta}}_{i}+\tau_{\mathrm{ex}, i}^{\phi} \hat{\boldsymbol{\phi}}_{i}$ and $\boldsymbol{\tau}_{\mathrm{bSTT}, i}=\tau_{\mathrm{bSTT}, i}^{\theta} \boldsymbol{\theta}_{i}+\tau_{\mathrm{bSTT}, i}^{\phi} \hat{\boldsymbol{\phi}}_{i}$. Finally, on the condition of a steady-state precession about the vertical axis, the damping torque on each spin must be along the local polar direction, i.e., $\boldsymbol{\tau}_{\mathrm{damp}, i}=\alpha \hat{\mathbf{m}}_{i} \times d \hat{\mathbf{m}}_{i} / d t=\tau_{\mathrm{damp}, i} \hat{\boldsymbol{\theta}}_{i}$. To clarify, in the forms $\boldsymbol{\tau}=\tau \hat{\boldsymbol{\theta}}$ and $\mathbf{T}=T \hat{\boldsymbol{\phi}}$, the scalar prefactors multiplying the unit vectors denote not vector magnitudes, $\tau \neq|\boldsymbol{\tau}|$ and $T \neq|\mathbf{T}|$, but rather the sign-inclusive component values, $\tau=\tau_{\theta}=\boldsymbol{\tau} \cdot \hat{\boldsymbol{\theta}}$ and $T=T_{\phi}=\mathbf{T} \cdot \hat{\boldsymbol{\phi}}$. Such a notation convention is used to avoid clustering of indices when possible.

It can be concluded that the net interfacial STT torque and the net interlayer exchange torque acting on the net magnetic moment $\boldsymbol{\mu}_{\text {net }}$ of the free layer can be written as $\mathbf{T}_{\mathrm{iSTT}}=T_{\mathrm{iSTT}} \hat{\boldsymbol{\theta}}_{1}$ and $\mathbf{T}_{\text {iex }}=T_{\text {iex }} \hat{\boldsymbol{\phi}}_{N}$. With respect to the net magnetic moment, however, these and remaining net torques take the general form $\mathbf{T}=T_{\mu} \hat{\boldsymbol{\mu}}_{\text {net }}+T_{\theta} \boldsymbol{\theta}_{\text {net }}+T_{\phi} \boldsymbol{\phi}_{\text {net }}$, where $T_{\mu} \hat{\boldsymbol{\mu}}_{\text {net }}$ denotes the radial component tending to increase or decrease the magnitude of $\boldsymbol{\mu}_{\text {net }}=\mu_{\text {net }} \hat{\boldsymbol{\mu}}_{\text {net }}$. Nevertheless, in specific cases, a given individual torque may have only one finite component, as illustrated below. Finally, we note that during steady-state precession about the vertical axis, when the magnitude $\boldsymbol{\mu}_{\text {net }}$ is unchanging, the vectorial sum of all net torques must be of the form $\mathbf{T}_{\text {net }}=\mathbf{T}_{\mathrm{iSTT}}+\mathbf{T}_{\mathrm{bSTT}}+\mathbf{T}_{\text {iex }}+\mathbf{T}_{\mathrm{damp}}=T_{\text {net }} \hat{\boldsymbol{\phi}}_{\text {net }}$, indicating a compensation of torque components, to be addressed in the coming analysis.

\section{STNO CHARACTERISTICS}

\section{A. In the absence of bulk STT: case 0}

First, we consider current-induced DW oscillations in an all-perpendicular spin-valve structure in the absence of bulk STT (case 0, Fig. 1a). This provides a clearer understanding of the effects of the interfacial STT on oscillator dynamics and the coupling between the polar and azimuthal degrees of freedom of the spin chain. The bulk STT can generally be neglected when the spin polarization of the electric current passing through the free layer is sufficiently small. A heterostructured free layer, for example, can be optimized for large STT near the free-layer/spacer interface and reduced STT within the bulk through appropriate materials selection.

We assume the soft free layer to be perfectly soft, i.e., to have zero magnetocrystalline anisotropy. The operation of the considered STNO is analyzed in the absence of an applied magnetic field. Oersted and magnetostatic fields are excluded from the model. Since we are considering an all-perpendicular spin-valve structure, the polarizer and the pinned layer are taken to be magnetized vertically. We chose the former to be magnetized up, i.e., $\hat{\mathbf{p}}=\left(\begin{array}{lll}0 & 0 & 1\end{array}\right)$, and the latter to be magnetized down, i.e., $\hat{\mathbf{q}}=\left(\begin{array}{lll}0 & 0 & -1\end{array}\right)$. In the absence of bulk STT and for a symmetric interfacial-STT efficiency Eq. (A5), the response of the modeled STNO is identical regardless of the relative orientations of the polarizer and pinner-layer magnetizations. 
(a)

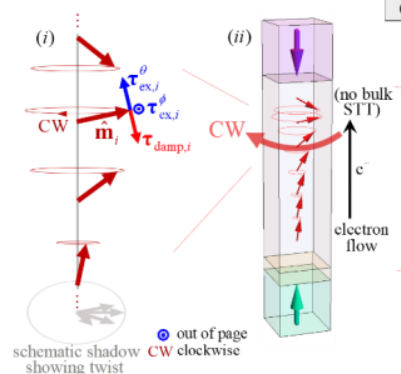

(b)

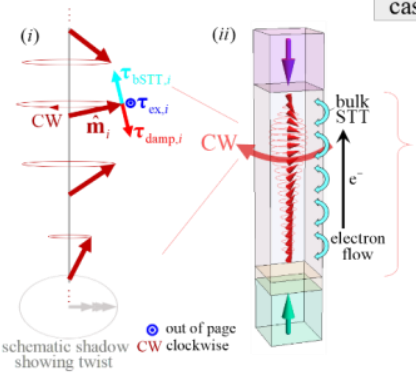

case 0

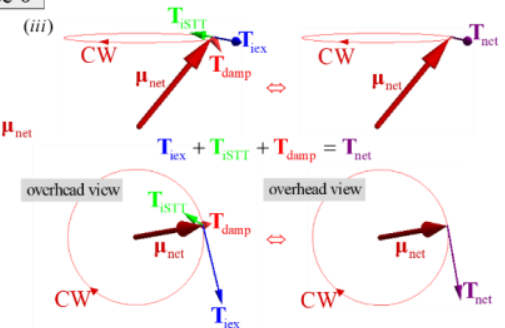

case 1
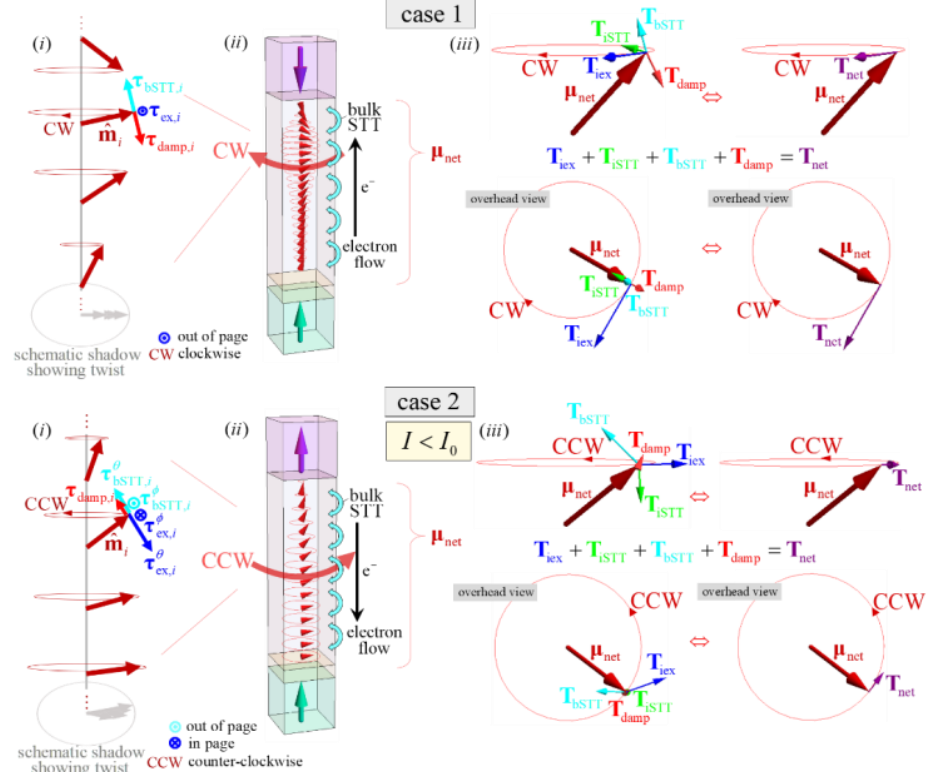

(d)

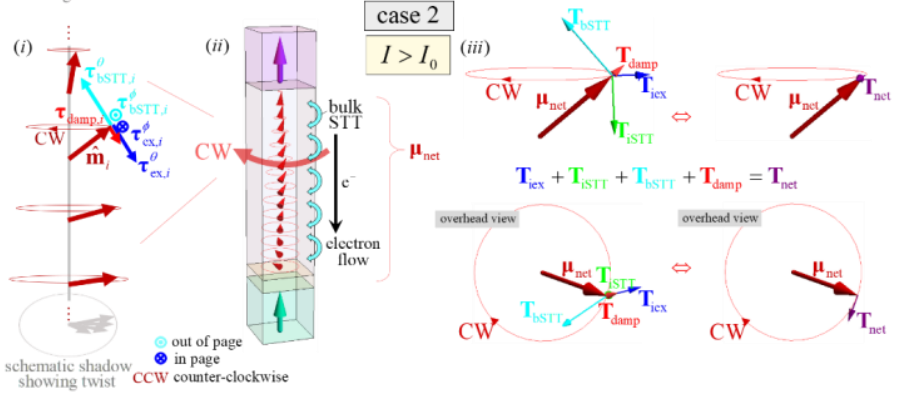

Figure 4: Illustrations of the competitions between local torques $\boldsymbol{\tau}_{i}$,s acting on an arbitrary interior spin $\hat{\mathbf{m}}_{i}$, and of net torques $\mathbf{T}$,s acting on the net free-layer magnetic moment $\boldsymbol{\mu}_{\text {net }}$, during steady state $\mathrm{CW}$ precession for cases 0,1 , and 2 . 
By symmetry, we expect the precession of spins of the free layer under an applied current to be about the vertical axis, as illustrated in Fig. 1a.

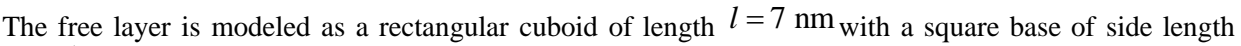
$w=5 \mathrm{~nm}$. The free-layer exchange length, saturation magnetization, and damping parameter are chosen to be $A_{\mathrm{ex}}=1.0 \mu \mathrm{erg} / \mathrm{cm}, M_{\mathrm{s}}=800 \mathrm{emu} / \mathrm{cm}^{3}$, and $\alpha=0.02$, respectively. The finite-differences cell size associated with each spin is $\Delta=1 \mathrm{~nm}$, hence $N=l / \Delta=7$ spins discretize the free layer magnetization. The energy density of the interlayer exchange coupling between the free and pinned layers is set to $J_{\mathrm{ex}}=2 A_{\mathrm{ex}} / \Delta$, which specifies an interlayer exchange coupling of the same strength as the exchange coupling between neighboring spins within the free layer. The device-specific constants in Eq. (A5) are chosen to be $q_{\mathrm{p}}=\AA=1$ and $q_{\mathrm{n}}=B=0$, so that $\eta(\theta)=1$. By numerically integrating the extended LLG Eq. (1), we obtain the dynamics of the spin chain describing the free-layer magnetization. Figure Erreur ! Source du renvoi introuvable.a shows the frequency of steady-state oscillations of the free-layer magnetization as a function of applied current. Electron flow in the direction from polarizer toward to the pinned layer is regarded as positive current in the present case.

At zero applied current, the system is in a stable equilibrium state with the free-layer magnetization oriented in the direction of the pinned layer (Fig. Erreur ! Source du renvoi introuvable.d(i)). The bulk and interlayer exchange energies are both minimum for this configuration. As the applied DC current is increased, the interfacial STT acts to tilt the bottommost spin of the free layer in the direction of the polarizer magnetization, i.e., counter to the magnetization of the pinned layer (Fig. Erreur ! Source du renvoi introuvable.d (ii)), provided there is a small perturbation of the bottommost spin that directs it away from its perfect alignment with the magnetization of the polarizer. With initial tilting of the first spin, the remaining spins in the chain tilt as well, owing to the bulk exchange interaction. As a result, a finite angle $\theta_{N \mathrm{q}}$ develops between the last spin $i=N$ in the chain and the magnetization direction of the pinned layer $\hat{\mathbf{q}}$. The finite angle $\theta_{N \mathrm{q}}$ implies an interlayer exchange torque exerted by the pinned layer on the free layer.

This torque, received by the topmost spin of the chain, is distributed over the remaining spins via the bulk exchange interaction and generates the precession of the free-layer magnetization as a whole.

Simulations show that the magnetization oscillations persist only if a current is applied in excess of a threshold value, which, for the specified STNO, is $I_{\text {th }} \approx 0.02 \mathrm{~mA}$. For $I<I_{\text {th }}$, the precessing magnetization relaxes back to its perfectly vertical equilibrium configuration (Fig. Erreur ! Source du renvoi introuvable.b(i)), regardless of the magnitude of the perturbation. A finite threshold current for sustained magnetization oscillations is a common feature of many STNO devices ${ }^{29,40,79}$.

From the above considerations, it is reasonable to expect that an increase in applied current leads to an increase in the angle $\theta_{N \mathrm{q}}$ between the topmost spin and the pinned-layer magnetization, and hence results in a greater interlayer exchange torque, implying faster precession. For relatively low currents, this is indeed the case, as implied by the initial positive correlation between the absolute frequency and applied current shown in the inset of Fig. Erreur ! Source du renvoi introuvable.a. Surprisingly, with greater increases in applied current, the frequency begins to level off and subsequently decrease (inset of Fig. Erreur: Source du renvoi introuvable.a), approaching asymptotically zero in the high current limit, i.e., $f \rightarrow 0$ as $I \rightarrow \infty$ (Fig. Erreur ! Source du renvoi introuvable.a). 


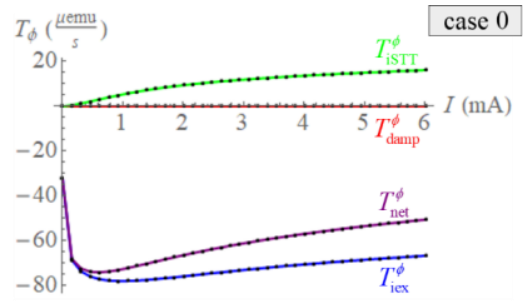

(a)

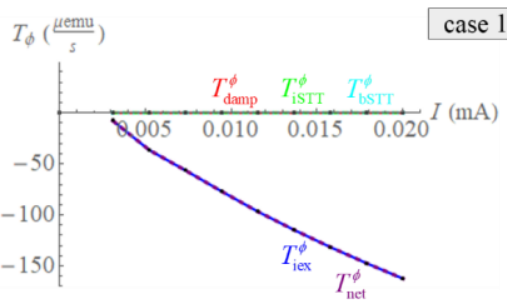

(b)

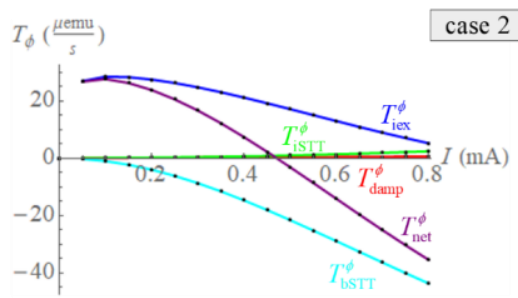

(c)

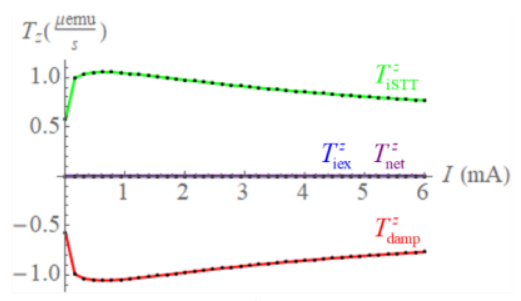

(d)

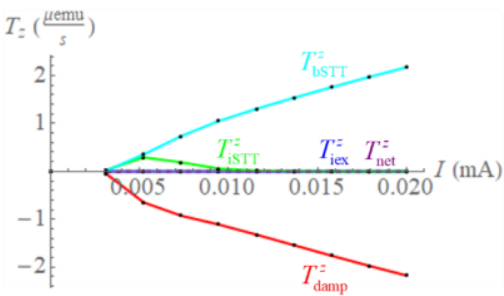

(e)

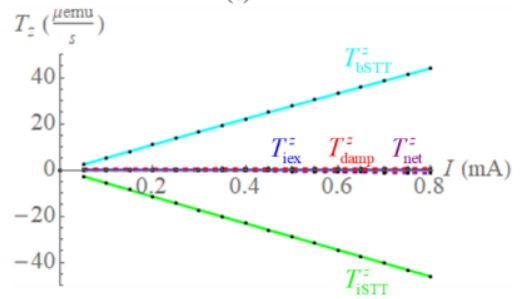

(f)

Figure 5: (a-c) Azimuthal and (d-f) vertical projections of the net interlayer exchange, interfacial spin-transfer, and damping torques, and the resultant net torque acting on the net free layer magnetic moment in cases 0,1 , and 2, given as a function of applied current. The azimuthal torque components drive STNO oscillations, while the vertical torque components mutually compensate for steady-state magnetization precession.

As noted, for zero applied current, the free-layer magnetization is stationary, with all spins oriented along the direction of the pinned-layer magnetization, i.e., ${ }^{\theta_{i}}=0$, for all $i$. As the current is initially increased, the spins tilt, and the polar angle of each spin becomes finite, i.e., $\theta_{i} \neq 0$. Simulations show that the twisting of the spin chain for sufficiently reduced current is relatively small, hence we can regard the spin chain as being approximately structurally planar at low currents. In this case (Fig. Erreur ! Source du renvoi introuvable.a,d(ii)), the azimuthal directions are nearly the same for all spins, i.e., $\hat{\phi}_{\text {net }} \equiv \hat{\phi}_{1} \approx \hat{\phi}_{2} \approx \ldots \approx \hat{\phi}_{N}$. Furthermore, the polar directions of all spins for low currents are mutually comparable as well, i.e., $\hat{\boldsymbol{\theta}}_{\text {net }} \equiv \hat{\boldsymbol{\theta}}_{1} \approx \hat{\boldsymbol{\theta}}_{2} \approx \ldots \approx \hat{\boldsymbol{\theta}}_{N}$. We, therefore, conclude that at low current bias, the interfacial STT, $\mathbf{T}_{\mathrm{iSTT}}=T_{\mathrm{iSTT}} \hat{\boldsymbol{\theta}}_{\text {net }}$, which acts in the $\hat{\boldsymbol{\theta}}_{\text {net }}$ direction, is balanced by the damping torque, 
$\mathbf{T}_{\text {damp }}=T_{\text {damp }} \hat{\boldsymbol{\theta}}_{\text {net }}$, of opposite sense, so as to result in steady-state precession of the spin chain about the vertical axis, i.e.,

$$
T_{\mathrm{iSTT}}+T_{\mathrm{damp}} \approx 0 \quad \text { (for low currents) } \text {. }
$$

The situation described by the equation above is analogous to that characterizing many previously proposed STNO designs $\mathbf{s}^{3,34,39,80,81}$. The interlayer exchange torque, $\mathbf{T}_{\text {iex }}=T_{\text {iex }} \hat{\phi}_{\text {net }}$, acting in the azimuthal direction $\hat{\phi}_{\text {net }}$, consequently, generates alone the precession of magnetization in the low current regime. Since, according to Eq. (A11), $T_{\text {iex }}<0$ for a downward-oriented pinned-layer magnetization $\left(q_{z}=-1\right.$ ), the magnetization precession of the free layer in the case considered is in the $\mathrm{CW}$ direction.

For a larger current bias, a twist in the spin chain becomes prominent (Fig. 4a(i) (shadow), Fig. 6a,d) and Eq. (4) no longer holds. That the spin chain necessarily features a twist during steady-state oscillations can be recognized by inspecting the conditions for steady-state precession of any interior spin of the chain (Fig. $4 \mathrm{a}(\mathrm{i}))$. If the spin chain is undergoing steady-state precession about the vertical axis as a whole, then each spin must be undergoing steady-state precession about the same axis individually. The damping torque, $\boldsymbol{\tau}_{\mathrm{damp}, i}=\alpha \hat{\mathbf{m}}_{i} \times d \hat{\mathbf{m}}_{i} / d t$, acting on an arbitrary interior spin ${ }^{i}$, consequently, acts in the $\hat{\boldsymbol{\theta}}_{i}$ direction in the case of steady-state $\mathrm{CW}$ precession about the vertical axis. For such precession to be possible, there must be an equal and opposite torque to balance $\boldsymbol{\tau}_{\mathrm{damp}, i}=\tau_{\mathrm{damp}, i} \boldsymbol{\theta}_{i}$. In our reduced complexity model, this torque could only come from the bulk exchange interaction, provided there exists a twist in the spin chain to generate it. A twist in the spin chain, therefore, is a necessary condition for steady-state precession of the free-layer magnetization in case 0 .

To restate the physics in simple terms, the nonuniform fanning of the spin chain (which specifies the $\theta_{-}$ profile) results in an uncompensated, azimuthal component of the bulk exchange torque, $\boldsymbol{\tau}_{\mathrm{ex}, i}^{\phi}=\tau_{\mathrm{ex}, i}^{\phi} \boldsymbol{\phi}_{i}$, on each interior spin, which generates its precession about the vertical axis, whereas the twisting of the spin chain (which specifies the ${ }^{\phi}$-profile) gives rise to the polar component of the bulk exchange torque, $\boldsymbol{\tau}_{\mathrm{ex}, i}^{\theta}=\tau_{\mathrm{ex}, i}^{\theta} \hat{\boldsymbol{\theta}}$, needed to balance the damping torque, $\boldsymbol{\tau}_{\mathrm{damp}, i}=\tau_{\mathrm{damp}, i} \hat{\boldsymbol{\theta}}_{i}$, so that steady-state precession is achieved. 


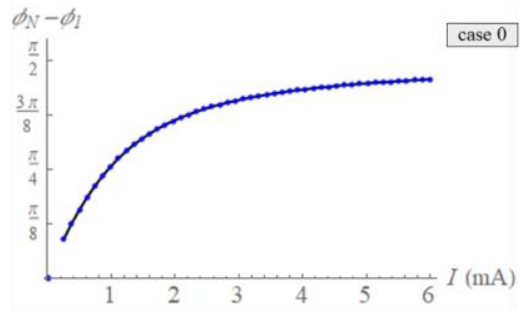

(a)

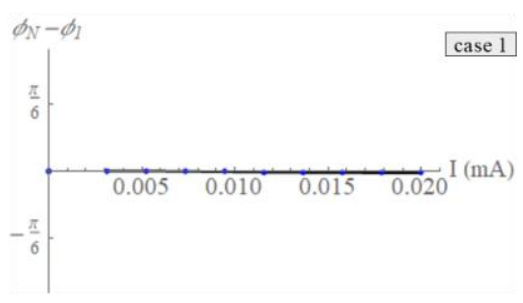

(b)

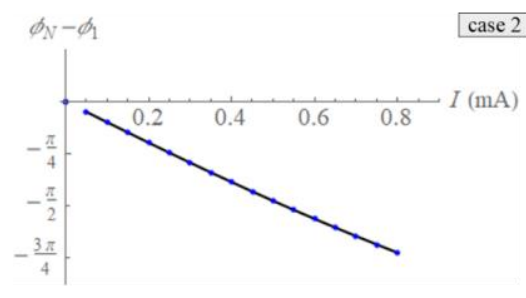

(c)

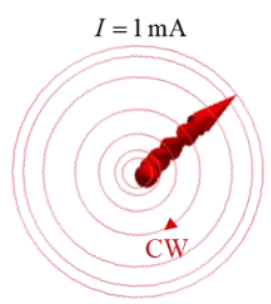

(d)

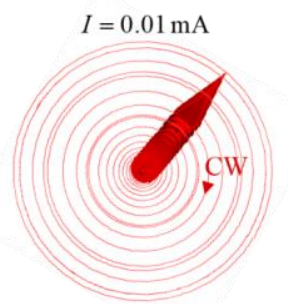

(e)

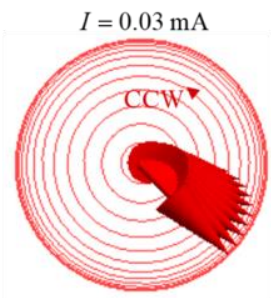

(f)

Figure 6: (a-c) The azimuthal angle difference, $\phi_{N}-\phi_{1}$, between the topmost and the bottommost free-layer spin as a function of applied current for cases 0,1 , and 2 . The angle difference reflects the twist in the spin chain, which monotonically changes with applied current in cases 0 and 2, while it is absent in case 1. (d-f) An overhead view of the free layer spins at a given current showing the developed twist in the spin change. (A careful comparison of the positions of the arrow tips with respect to their circular traces reveals that in case 0 $\phi_{N}-\phi_{1} \approx 45^{\circ}$ for the specified current value.)

In the continuum limit, a finite $\boldsymbol{\tau}_{\mathrm{ex}}^{\psi}$ implies a non-vanishing second derivative of the polar angle ${ }^{\theta(z)}$, whereas a finite $\boldsymbol{\tau}_{\mathrm{ex}}^{\theta}$ implies a non-vanishing first derivative of the azimuthal angle $\phi(z)$ with respect to the longitudinal coordinate $z^{50}$. Since the rate of precession and, consequently, $\boldsymbol{\tau}_{\text {damp initially increase }}$ with current, the counterbalancing torque component $\boldsymbol{\tau}_{\mathrm{ex}}^{\theta}$ must also increase for steady-state precession to 
persist at larger applied current. This implies increased twist at larger current amplitudes and oscillation frequencies (Fig. 6a).

Since the azimuthal direction $\hat{\phi}_{i}=\left(\begin{array}{lll}-\sin \phi_{i} & \cos \phi_{i} & 0\end{array}\right)$ of each spin is substantially different in the presence of prominent twist, Eq. (4) no longer holds, as it was based on the approximation of structural planarity of the spin chain. Figure $4 \mathrm{a}$ (iii) demonstrates that the net interfacial STT, $\mathbf{T}_{\mathrm{iSTT}}=T_{\mathrm{iSTT}} \boldsymbol{\theta}_{1}$, is no longer in balance with the net damping torque, $\mathbf{T}_{\text {damp }}$, since the two torques in the presence of prominent twist have significantly different directions.

Along the vertical direction, the net damping torque, $\mathbf{T}_{\text {damp }}$, is still compensated by $\mathbf{T}_{\text {iSTT }}$ (Figs. 4a(iii) and $5 \mathrm{~d})$, so that steady-state precession is realized:

$$
T_{\mathrm{iSTT}}^{z}+T_{\mathrm{damp}}^{z}=0
$$

The net torque generating the precession of the twisted spin chain, therefore, is equal to the vectorial sum of the interlayer exchange torque, $\mathbf{T}_{\text {iex }}$, and the uncompensated component of the interfacial STT, $\mathbf{T}_{\text {iSTT }}+\mathbf{T}_{\text {damp }}$. The uncompensated STT component given by the latter sum is necessarily an in-plane component, such that the net torque

$$
\mathbf{T}_{\text {net }}=\mathbf{T}_{\text {iex }}+\mathbf{T}_{\mathrm{iSTT}}+\mathbf{T}_{\mathrm{damp}}
$$

is along the azimuthal $\hat{\boldsymbol{\phi}}_{\text {net }}$ direction of the net magnetic moment $\boldsymbol{\mu}_{\text {net }}$, i.e., $\mathbf{T}_{\text {net }}=T_{\text {net }} \hat{\boldsymbol{\phi}}_{\text {net }}$, as required for steady-state precession about the vertical axis (Fig. 4a(iii)).

As can be seen in Fig. 4a(iii), the in-plane component of the net interfacial STT projects negatively onto the azimuthal component of the net interlayer exchange torque, thus exerting a braking action that slows down the precession of the free-layer magnetic moment. Geometrically, as the twist increases with increasing current (Fig. 6a), the net interfacial STT orients more in the direction opposite to that of the net interlayer exchange torque, bringing about a reduction of oscillation frequency with increasing current, as observed in Fig. Erreur ! Source du renvoi introuvable.a for high current values. The competition between the azimuthal components of the net torques and the compensation of their vertical components is shown in Figs. 5a and 5d.

In the limit $I \rightarrow \infty$ the oscillation frequency is observed to approach zero (Fig. Erreur ! Source du renvoi introuvable.a). This implies static equilibrium in the high-current limit. Such an equilibrium is only possible on condition that $T_{\text {net }}=0$. Indeed, as simulations reveal, the azimuthal-angle difference between the first and last spin in the twisted chain approaches $90^{\circ}$ in the given limit, i.e., $\phi_{N}-\phi_{1} \rightarrow 90^{\circ}$ as $I \rightarrow \infty$

(Fig. 6a). According to Eqs. (A10) and (A11), in this limit, the interfacial STT tends toward an antiparallel alignment with the interlayer exchange torque, as shown in Fig. 7, which results in torque cancellation ( $T_{\text {net }} \rightarrow 0, f \rightarrow 0$ ).

It can be intuitively argued that in the high current limit steady-state oscillations cannot persists in case 0 . Namely, when $I \rightarrow \infty$, we expect that $\mathbf{m}_{1} \rightarrow\left(\begin{array}{lll}0 & 0 & -1\end{array}\right)$ on account of an infinitely coercive interfacial STT. A vertically oriented spin, however, cannot precess about the vertical axis, as it possesses no in-plane 

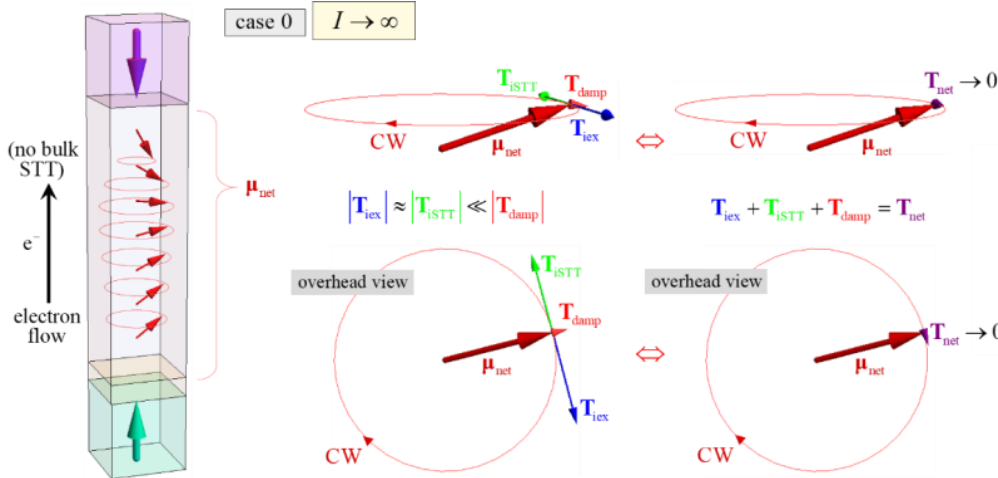

$\boldsymbol{\mu}_{\text {net }}$

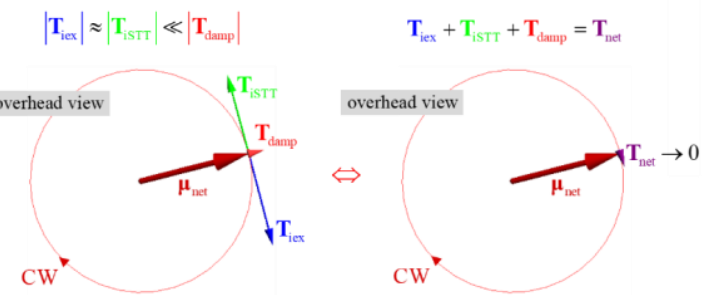

Figure 7: Illustrations of the net magnetic moment of the free layer, ${ }^{\boldsymbol{\mu}_{\text {net }}}$, and the torques acting on it in case 0 when $I \rightarrow \infty$. In the high-current limit, the net interfacial STT, $\mathbf{T}_{\mathrm{iSTT}}$, counterbalances the action of the net interlayer exchange torque, $\mathbf{T}_{\text {iex }}$, thus bringing the system into a state of static equilibrium. As Fig. 6a shows, the twist in the spin chain (which in the considered limit is confined to the bottommost region of the free layer) approaches $90^{\circ}$, and thus, according to (A10) and (A11), $\mathbf{T}_{\text {iSTT }}$ tends toward an antiparallel alignment with $\mathbf{T}_{\text {iex }}$

component; hence, it cannot transmit power to the neighboring spin in the chain through the exchange interaction.

In effect, the limiting condition $I \rightarrow \infty$ is equivalent to the condition of a vertically fixed bottommost spin. Since the magnetic moment of the pinned layer is also vertically fixed, the system reduces to a string of spins with vertically fixed ends, a basic micromagnetics boundary-value problem with a well-known equilibrium solution. This solution consists of a DW centered at the midpoint between the two boundaries, with no twist and a constant rate of change of the polar angle $\theta$ along the direction of the string of spins, i.e., uniform fanning ${ }^{82}$. This is precisely the solution obtained by our simulations for the spin valve in the high current limit (Fig. 2d(v)), where the $\phi_{N}-\phi_{1} \rightarrow 90^{\circ}$ difference between the first and last spin of the chain in the high-current limit is reconciled with the no-twist solution of the basic micromagnetic boundaryvalue problem by virtue of the fact that the first (bottommost) spin of the spin chain has a vanishing inplane component, i.e., $\theta_{1} \rightarrow 0$ as $I \rightarrow \infty$. As Fig. 6d shows, even for a relatively low current, the twisting of the spin chain is concentrated near the bottom of the free layer where the spins are largely vertically oriented, so the twist is not very noticeable. In the continuum limit $(N \rightarrow \infty)$, when $I \rightarrow \infty$, the twisting is confined to an infinitesimally small region at the base of the free layer, and since $\theta(z=0) \rightarrow 0$, the overall spin configuration can equivalently be regarded as having no twist.

Considering that steady-state precession in case 0 depends sensitively on the azimuthal angle difference $\phi_{N}-\phi_{1}$, which is highly affected by fluctuations of spin direction, we expect considerable spectral linewidth broadening in the presence of thermal agitation of the spins due to finite ambient temperature and Joule heating. The stochastic fluctuations of the direction of the bottommost spin of the free layer imply not only fluctuations of the interfacial-STT magnitude, but also its direction. A drastic effect of thermal fluctuations is expected at larger currents, when the bottommost free-layer spin is mostly vertically oriented 
$\left(\theta_{1} \approx 0\right)$ and $\mathbf{T}_{\text {iSTT }}$ is large. Under such conditions, a very small fluctuation of the spin's direction can result in a diametrical change of the torque's orientation. Since $\mathbf{T}_{\text {iSTT }}$ drives oscillations by forcing a DW into the free layer wherein it precesses about the interlayer exchange field, and modulates the precessional frequency through its braking action, thermal fluctuations at larger currents theoretically are expected to highly impact the oscillator's dynamical response in case 0 .

It must be noted, however, that for the present STNO specifications, a current of $I=1 \mathrm{~mA}$, closely matching the amplitude at which the frequency of oscillations is maximum (Fig. 2a), corresponds to a current density of $j=2.78 \times 10^{9} \mathrm{~A} / \mathrm{cm}^{2}$, which exceeds by roughly two orders of magnitude the breakdown threshold for typical spin-valve devices. It would, therefore, be experimentally difficult to study the oscillator response in the high-current regime in case 0 , as the device would likely not be able to withstand the extreme conditions of operation. Exploring the physics of the frequency dependence on current in case 0 , nonetheless highlights the competition between the participating torques and the role of DW twist, which is particularly useful in explaining the behavior of the STNO under case-1 and case-2 operating conditions discussed in the subsequent sections.

The effects of damping and other material and structural parameters on the STNO dynamical response in case 0 are presented in the Supplementary Material.

\section{B. In the presence of bulk STT: cases 1 and 2}

We investigate the current-frequency characteristics of a spin valve of the same structural and material properties as described previously, except now we consider the current passing through the free layer to be spin polarized by setting, for the first set of simulations, $P=1$ in Eq. (A7). This leads to the emergence of the bulk STT, which distinctly affects the STNO response. Later we shall inspect the dependence of the $f-I$ characteristics on the value of the spin polarization $P$. To simplify analysis, we shall ignore nonadiabatic effects, and thus impose $\beta=0$ in Eq. (A6). (Simulations with $\beta \sim \alpha$ indicated that the nonadiabatic effects on dynamics of our oscillator were not significant.)

Figures $2 \mathrm{~b}$ and $2 \mathrm{c}$ show that in the presence of bulk STT, the $f-I$ characteristics of the modeled STNO are qualitatively different for the two considered operating configurations, i.e.: (case 1) when the magnetic moments of the polarizer and the pinned layer are oriented antiparallel to each other (Fig. 1b), and (case 2) when they are oriented parallel to each other (Fig. 1c). To induce oscillations, the electric current in case 1 must be applied so that electrons flow from the pinned layer toward the polarizer, while in case 2, the electric current should be applied in the opposite direction; otherwise the applied current stabilizes the spins in a static vertical configuration.

\section{Case 1}

The bulk STT tends to translate a DW in the direction of the electron flow. In case 1, the spin current passing through the free layer pushes the DW against the pinned layer, causing it to compress (Fig. 2e). This compression of the DW results in an increased bulk exchange torque in the azimuthal direction, and hence increased rate of precession ${ }^{47,50}$. Since the DW cannot exit the free layer at the top interface because the pinned-layer magnetization is fixed, increased current inevitably implies increased DW compression and greater oscillation frequency (Fig. 2b). 
Simulation results presented in this section where obtained using a spin-chain model consisting of $N=21$ spins (cell size $\Delta=0.33 \mathrm{~nm}$ ) in order to resolve the spatial magnetization variation associated with DW compression against the pinned layer in case 1 (Fig. 2e).

To reach a deeper understanding of the interplay between the different participating interactions resulting in steady-state precession in case 1 , we inspect the balance of torques necessary for the steady-state precession of an arbitrary spin interior to the free-layer spin chain. In Fig. 4b(i), we see that the bulk STT, $\boldsymbol{\tau}_{\mathrm{bSTT}, i}=\tau_{\mathrm{bSTT}, i}, \boldsymbol{\theta}$

the damping torque, $\boldsymbol{\tau}_{\mathrm{damp}}=\tau_{\mathrm{damp}} \hat{\boldsymbol{\theta}}_{i}$. Simulation results confirm that these two torques indeed compensate one another for each interior spin of the chain during steady-state precession, i.e., $\boldsymbol{\tau}_{\mathrm{bSTT}, i}+\boldsymbol{\tau}_{\mathrm{damp}, i}=\mathbf{0}$, for all $i$. A twist in the spin chain, therefore, is not a necessary condition for steady-state precession in the presence of bulk STT for the considered configuration (case 1, Fig. 4b(ii)), as the damping torque $\boldsymbol{\tau}_{\text {damp, } i}$ is already balanced at each interior spin by $\boldsymbol{\tau}_{\mathrm{bSTT}, i}$.

Simulations confirm that a twist does not develop during steady-state oscillations in case 1 (Fig. 6b). Still, as in the absence of bulk STT (case 0, Fig. 4a(i)), it is the bulk exchange torque, $\boldsymbol{\tau}_{\mathrm{ex}, i}=\tau_{\mathrm{ex}, i} \boldsymbol{\phi}_{i}$, arising from the nonuniform fanning of the spins that generates the precession in the azimuthal direction internally.

Externally, as required for steady-state precession about the vertical axis, the vertical components of the torques acting on the net magnetic moment balance (Figs. $4 \mathrm{~b}$ (iii) and 5e),

$$
T_{\mathrm{iSTT}}^{z}+T_{\mathrm{bSTT}}^{z}+T_{\mathrm{damp}}^{z}=0
$$

The net torque, equal to the vectorial sum

$$
\mathbf{T}_{\text {net }}=\mathbf{T}_{\mathrm{iex}}+\mathbf{T}_{\mathrm{iSTT}}+\mathbf{T}_{\mathrm{bSTT}}+\mathbf{T}_{\mathrm{damp}}
$$

acts, in consequence of Eq. (7), in-plane and orthogonal to $\boldsymbol{\mu}_{\text {net }}$, i.e., in the azimuthal $\hat{\boldsymbol{\phi}}_{\text {net }}$ direction, thus generating precession of the net free-layer magnetic moment about the vertical axis. Schematically this is illustrated in Fig. $4 b$ (iii).

Figure $5 \mathrm{~b}$ shows that for large enough applied currents, the interfacial STT is negligibly small in comparison to the remaining torques in equation Eq. (8). This can be expected in view of the near-perfect vertical orientation of the bottommost spin $(i=1)$ for such operating conditions. Under such conditions, the described spin valve responds equivalently to the STNO investigated by Franchin, et al. ${ }^{50}$, involving a free layer pinned at both ends, and an absence of interfacial STT. The authors have analytically determined the $f-I$ characteristics for such a system in the low and high current regimes. Our simulations similarly show that for large current amplitudes the frequency is proportional to the square of the current, i.e., $|f| \propto I^{2}$.

For a discussion of the dependence of frequency on the damping constant and other material and structural parameters, see the Supplementary Material.

\section{Code de champ modifie}




\section{Case 2}

Qualitatively very different $f-I$ characteristics are obtained for case 2 , when the polarizer and pinnedlayer magnetizations are oriented in the same direction, and the electrons stream from the pinned layer toward the polarizer. For convenience, we shall redefine here positive current to correspond to electron flow from the pinned layer toward the polarizer. Simulations of spin-chain dynamics in case 2 were obtained using a spin chain involving $N=14$ spins (cell size $\Delta=0.5 \mathrm{~nm}$ ). Figure 2c shows for the described operating configuration a threshold current of $I_{\text {th }} \approx 0.05 \mathrm{~mA}$ required for sustained CCW magnetization precession. From this mark ( $f \approx 40 \mathrm{GHz}$ ), the oscillation frequency is seen to gradually decrease to zero with increasing current amplitude, at which point the DW magnetization ceases to precess $(f=0)$. As current is increased further, precession commences again, but now in the opposite CW direction $(f<0)$.

For the material and structural specifications of the STNO described, the current amplitude at which static equilibrium is reached $\left(I_{0} \approx 0.5 \mathrm{~mA}\right.$; Fig. $\left.2 \mathrm{c}\right)$ in case 2 corresponds to a current density of $j_{0}=1.4 \times 10^{9} \mathrm{~A} / \mathrm{cm}^{2}$ straightforward, therefore, to experimentally explore the STNO response within a current range inclusive of $I_{0}$. Yet, we do not dismiss the possibility that certain modifications to STNO specifications may result in a sufficient reduction of the transition current, ${ }{ }_{0}$, that would make experimental investigation of the oscillator response within its vicinity feasible. Explaining the unique frequency-current characteristic shown in Fig. 2c, in any event, proves to be useful in broadening our understanding of the nature of possible torque competitions in spin-valve devices featuring a DW.

For a deeper insight into the physics responsible for the atypical oscillator behavior illustrated in Fig. 2c, we turn our attention to the torques exerted on an arbitrary interior spin within the free-layer spin chain. For low current values, precession of the net magnetic moment is predominantly due to the interlayer exchange torque supplied by the pinned layer (Fig. 5c). Since the pinned layer in case 2 is oriented upward, the precession is initially in the CCW direction (or sense) when viewed from the top. The sense of magnetization precession specifies the orientation of the damping torque, $\boldsymbol{\tau}_{\mathrm{damp}, i}=\alpha \mathbf{m}_{i} \times \dot{d} \hat{\mathbf{m}}_{i} / d t$, which is proportional to the first time-derivative of the magnetization.

Figure 4c(i) illustrates the torques acting on an arbitrary interior spin when the magnetization precession is in the $\mathrm{CCW}$ direction. For current values giving rise to $\mathrm{CCW}_{\theta}$ precession, the damping torque is oriented in the ${ }^{-\hat{\boldsymbol{\theta}}_{i}}$ direction. The polar component of the bulk STT, $\boldsymbol{\tau}_{\mathrm{bSTT}, i}^{\theta^{\mathrm{P}}}$, is also oriented in the ${ }^{-\hat{\boldsymbol{\theta}}_{i}}$ direction, as it tends to propel the $\mathrm{DW}$ in the direction of the electron flow, i.e., toward the polarizer. These two torques, $\boldsymbol{\tau}_{\mathrm{damp}, i}=\tau_{\mathrm{damp}, i} \boldsymbol{\theta}_{i}$ and $\boldsymbol{\tau}_{\mathrm{bSTT}, i}^{\theta}$, therefore, constructively superimpose. Yet, we know that for steady-state precession the resultant polar torque on each spin must vanish. We thus conclude that a twist in the spin chain is a necessary condition for steady-state CCW precession in the presence of bulk STT in case 2, so that torque compensation in the polar direction is achieved, i.e., $\boldsymbol{\tau}_{\mathrm{damp}, i}+\boldsymbol{\tau}_{\mathrm{bSTT}, i}^{\theta}+\boldsymbol{\tau}_{\mathrm{ex}, i}^{\theta}=\mathbf{0}$, where $\boldsymbol{\tau}_{\mathrm{ex}, i}^{\sigma^{\prime}}$ is the polar component of the bulk exchange torque arising from the twist. Furthermore, we conclude that the 
sense of the twist is such that the bottommost spin leads in the CCW precession of the spin chain, i.e., $\phi_{1}>\phi_{2}>\ldots>\phi_{N}$, so that $\boldsymbol{\tau}_{\mathrm{ex}, i}^{\theta}$ is oriented opposite to the remaining two torques, $\boldsymbol{\tau}_{\mathrm{damp}, i}$ and $\boldsymbol{\tau}_{\mathrm{bSTT}, i}^{\theta}$.

Simulations confirm the presence of twist in the spin chain during steady-state CCW precession in case 2 (Fig. 6c,f).

The twist, which is a necessary condition for steady-state precession in the presence of bulk STT in case 2, implies the existence of a finite azimuthal component of the bulk STT, ${ }^{\phi}{ }_{\mathrm{bSTT}, i}$. For the particular sense of twist inherent to steady-state CCW precession in case 2 (Fig. 6c), ${ }^{\boldsymbol{t}_{\mathrm{bSTT}, i}^{\phi}}$ is oriented counter to the azimuthal component of the bulk exchange torque, $\boldsymbol{\tau}_{\mathrm{ex}, i}^{\phi}$, resulting from the nonuniform fanning of the spins. Consequently, the azimuthal component of the bulk STT, $\boldsymbol{\tau}_{\mathrm{bSTT}, i}^{\phi}$, whose magnitude is monotonically increasing with applied current, exerts a breaking action upon the $\mathrm{CCW}$ precession of the free-layer magnetization.

With increasing current and twist, the azimuthal component of the bulk STT, ${ }_{\text {bSTT,i }}^{\phi}$, acting on each internal spin ${ }^{i}$, grows in magnitude, ultimately counter-balancing the azimuthal component of the bulk exchange torque, ${ }^{\boldsymbol{\tau}_{\mathrm{ex}, i}^{\phi}}$. At compensation, i.e., ${ }^{\boldsymbol{\tau}_{\mathrm{ex}, i}^{\phi}+\boldsymbol{\tau}_{\mathrm{bTT}, i}^{\phi}=\mathbf{0}}$, static equilibrium is reached, hence ${ } f\left(I_{0}\right)=0$, for some particular current value $I_{0}$ (Fig. 4c(i)). With further increases in current, $\boldsymbol{\tau}_{\mathrm{bSTT}, i}^{\phi}$ overcomes $\boldsymbol{\tau}_{\mathrm{ex}, i}^{\phi}$, i.e., $\left|\boldsymbol{\tau}_{\mathrm{bSTT}, i}^{\phi}\right|>\left|\boldsymbol{\tau}_{\mathrm{ex}, i}^{\phi}\right|$, and precession commences again, but now in the opposite, $\mathrm{CW}$ direction.

Figure $4 \mathrm{~d}(\mathrm{i})$ shows the torques acting on an interior spin when $I>I_{0}$, i.e., in the case of $\mathrm{CW}$ precession, where $\left|\boldsymbol{\tau}_{\mathrm{bSTT}, i}^{\phi}\right|>\left|\boldsymbol{\tau}_{\mathrm{ex}, i}^{\phi}\right|$. The damping torque, $\boldsymbol{\tau}_{\mathrm{damp}, i}$, being proportional to the time-rate of change of the spin direction vector $\hat{\mathbf{m}}_{i}$, changes orientation with the change of the sense of precession, and hence is now in the direction counter to the polar component of the bulk STT, $\boldsymbol{\tau}_{\mathrm{bSTT}, i}^{\theta}$ (Fig. 4d(i)). Since the twist of the spin chain increases continuously as the current approaches $I_{0}$ from below, it does not undergo a sudden change in sense as the current crosses $I_{0}$. Consequently, the azimuthal components of the bulk STT, $\boldsymbol{\tau}_{\mathrm{bSTT}, i}^{\phi}$ , and the bulk exchange torque, ${ }^{\boldsymbol{e}_{\mathrm{ex}, i},}$, do not change orientation upon the transition from $\mathrm{CCW}$ to $\mathrm{CW}$ precession. Simulation show that the twist continues increasing linearly as current is increased beyond $I_{0}$ (Fig. 6c).

From the viewpoint of net magnetic moment (Figs. 3c(iii) and $4 \mathrm{~d}(\mathrm{iii})$ ), the vertical components of the net torques balance, as expressed in Eq. (7), while the resultant torque $\mathbf{T}_{\text {net }}$, given by Eq. (8), necessarily acts in the $\hat{\phi}_{\text {net }}$ direction, thus generating magnetization precession about the vertical axis, similarly as in case 1 . However, unlike in case 1, the magnitude of $\mathbf{T}_{\text {net }}$ is not monotonically increasing with current, as implied by the ${ }^{f-I}$ characteristic shown in Fig. 2c, and as observed in Fig. 5c.

The main contribution to $\mathbf{T}_{\text {net }}$, when $I<I_{0}$, comes from the interlayer exchange torque $\mathbf{T}_{\text {iex }}$, as seen in Figs. 4c(iii) and 5c. The upward orientation of the pinned-layer magnetization means that precession occurs 
in the CCW direction when viewed from the top, according to Eq. (A11). Figures $4 \mathrm{c}$ (iii) and 5c show that the bulk STT, $\mathbf{T}_{\mathrm{bSTT}}$, counters $\mathbf{T}_{\text {iex }}$ along the azimuthal direction. With increasing current, $\mathbf{T}_{\mathrm{bSTT}}$ increasingly exerts a braking action on the magnetization precession, ultimately causing it to stop ( $f=0$ ) at $I=I_{0}$.

When the applied current $I$ exceeds $I_{0}$, the azimuthal component of $\mathbf{T}_{\mathrm{bSTT}}$ overcomes the azimuthal component of $\mathbf{T}_{\text {iex }}$, and precession commences again, only now in the opposite, CW direction when viewed from the top (Fig. 4d(iii)).

Neither for $I<I_{0}$ (Figs. 4e(iii)) nor for $I>I_{0}$ (Fig. 4d(iii)) is the azimuthal component of the interfacial STT, $\mathbf{T}_{\text {iSTT }}$, or the damping torque, ${ }_{\text {damp }}$, comparable to that of the azimuthal components of $\mathbf{T}_{\text {iex }}$ and $\mathbf{T}_{\text {bSTT }}$ (Fig. 5c). This remains so even for very large current, $I \square I_{0}$, when the magnitude of $\mathbf{T}_{\text {iSTT }}$ is substantial. This is because in case 2, the bottommost spin adjacent to the polarizer layer is oriented almost entirely in-plane (Fig. 2f), and hence ${ }^{\mathbf{T}_{\text {iSTT }}}\left({ }^{\perp \hat{\mathbf{m}}_{1}}\right)$ is almost perfectly vertical, i.e., it has no appreciable in-plane (azimuthal) component.

The half-DW-like spin configuration of the free layer observed in case 2 (Fig. 2f) reflects the competition between the interfacial and bulk STTs in displacing the magnetization nonuniformity within the free layer in the vertical direction: the interfacial STT tends to drive a DW into the free layer, while the bulk STT works to expel the DW at the bottom end of the free layer by pushing it downward, in the direction of the electron flow.

A discussion of the influence of damping and other STNO parameters on STNO response is provided in the Supplementary Material.

\section{CONCLUSION}

We investigated the spin-transfer-driven oscillations of a transverse domain wall confined to an allperpendicular spin-valve structure using a 1D micromagnetic model. The response of the STNO under three operating conditions was considered: (case 0 ) in the absence of spin polarization of the electron flow within the free layer (i.e., in the absence of bulk STT), and (cases 1 and 2) in the presence of bulk STT, when itinerant electrons flow from the polarizer toward the pinned layer (case 1), and when they flow in the opposite direction (case 2). It was found that the twist of the magnetization about the vertical axis of precession plays a key role in the operation of the studied STNO. It was shown that the twist develops as a necessary condition for steady-state oscillations about the vertical axis in case 0 and case 2 . As discussed in the Supplementary Material, owing to this twist, the frequency of oscillations is virtually insensitive to the damping constant $\alpha$ of the free layer in case 2 . This insensitivity is attributed to the nearly complete balance between the interfacial and the bulk spin-transfer torques in the vertical direction. Due to the competition between these two torques in the azimuthal direction, the precession of the free-layer magnetization is found to switch from $\mathrm{CCW}$ to $\mathrm{CW}$ as the applied current is raised beyond a certain compensation-point value.

We showed the way in which the material and structural parameters influence the frequency of the STNO in the three cases considered and suggested how variations in the values of these parameters may affect the spectral linewidth (Supplementary Material). The highly atypical dynamical response of our STNO to 
current for the three cases investigated and the different competitions that arise in each case between the acting torques suggest that the described STNO may be found useful for fundamental investigations of spintronic and magnetic phenomena at the nanoscale. The nature of the interplay between the exchange, STT, and damping torques, resulting in qualitatively different STNO characteristics obtained for cases 0-2 (Fig. 4a-c), suggests that introducing additional interactions, such as SOT or Dzyaloshinskii-Moriya interaction (DMI), in a confined current-driven system such as the one studied here, or extensions of it, may result in altered modes in which participating torque components cooperate in producing steady-state precession, which could sensitively affect STNO performance. Moreover, the dependence of twist on current, as observed in cases 0 and 2 (Fig. 6a, c), invites the question of how a spiral spin structure ${ }^{83-85}$ having inherent twist may interact with the spin-polarized current in the confines of a spin valve. The interaction between the two may result in the partial unwinding or further twisting of the magnetic structure, with a concomitant change in oscillation frequency. Exploring the operation of similar systems featuring additional interactions could thus reveal new possibilities for tailoring STNO response to current. The prospect of optimizing STNO response for reduced sensitivity to the damping constant, as indicated here, could have additional implications for spintronic-device engineering and related technologies.

While steady-state magnetization precession about the vertical axis in an all-perpendicular spin-valve structure, considered here, does not generate power output through the magnetoresistive effect, it does produce an oscillating stray magnetic field, which could be useful for microwave-assisted magnetic recording and for dipolar coupling between oscillators through the magnetostatic interaction. Alterations to the all-perpendicular STNO design, such as introducing a non-perpendicular anisotropy component to one or more of the layers ${ }^{41,86-89}$, or extensions to the STNO geometry, such as a transformation to a threeterminal device ${ }^{90,91}$, could be considered as a means for extracting microwave electrical signals from the oscillator, though such considerations are beyond the scope of the present work.

\section{ACKNOWLEDGEMENTS}

Work at UCSD was supported by Quantum-Materials for Energy Efficient Neuromorphic-Computing, an Energy Frontier Research Center funded by DOE, Office of Science, BES under Award DE-SC0019273. This work was also supported by the impact project LUE-N4S, part of the French PIA project "Lorraine Université d'Excellence", reference ANR-15IDEX-04-LUE, and by the "FEDER-FSE Lorraine et Massif Vosges 2014-2020", a European Union Program.

\section{APPENDIX: Spin-chain model details: field and torque calculations}

The exchange field within the 1D spin-chain model (Eq. (1)), is given by

$$
\mathbf{H}_{\mathrm{ex}, i}=\frac{2 A_{\mathrm{ex}}}{M_{\mathrm{s}} \Delta^{2}}\left(\hat{\mathbf{m}}_{i+1}+\hat{\mathbf{m}}_{i-1}\right)
$$

where $A_{\text {ex }}(\mathrm{erg} / \mathrm{cm})$ is the exchange stiffness constant. The thickness $\Delta(\mathrm{cm})$ denotes the portion of the length of the free layer occupied by each spin, i.e., $\Delta=l / N$, where $l$ is the free-layer thickness and $N$ is the number of spins discretizing the free-layer magnetization. A numbering convention is adopted such that the free-layer spin adjacent to the polarizer is regarded as the first spin in the chain $(i=1)$, while the free- 
layer spin adjacent to the pinned layer is regarded as the last spin $(i=N)$. We account for the discontinuity of the free layer at the top and bottom interfaces via the imposition $\hat{\mathbf{m}}_{0}=\hat{\mathbf{m}}_{N+1}=\mathbf{0}$.

The interlayer exchange field is due to the ferromagnetic interlayer coupling between the free layer and the pinned layer. This field is exerted only at the boundary, specifically, upon the topmost free-layer spin ( $i=N$ ), adjacent to the pinned layer. The expression for the interlayer exchange field is

$$
\mathbf{H}_{\mathrm{iex}, i}=\delta_{i N} \frac{\int_{\mathrm{ex}}}{M_{\mathrm{s}} \Delta} \hat{\mathbf{q}}
$$

where $\delta_{i N}$ is the Kronecker delta function, $J_{\text {ex }}\left(\mathrm{erg} / \mathrm{cm}^{2}\right)$ is the interlayer exchange-coupling energy density, and $\hat{\mathbf{q}}$ is the unit direction vector of the pinned-layer magnetization.

The uniaxial magnetocrystalline anisotropy field for the ${ }^{i \text { th }}$ spin is expressed as

$$
\mathbf{H}_{\text {anis }, i}=\frac{2 K_{\mathrm{u}}}{M_{\mathrm{s}}}\left(\hat{\mathbf{m}}_{i} \cdot \hat{\mathbf{k}}\right) \hat{\mathbf{k}}
$$

where $K_{\mathrm{u}}\left(\mathrm{erg} / \mathrm{cm}^{3}\right)$ and $\hat{\mathbf{k}}$ represent the free-layer magnetocrystalline-anisotropy energy density and the unit vector in the direction of the associated easy axis.

The interfacial STT, appearing in Eq. (1) as $\boldsymbol{\tau}_{\text {interface, } i}$, is modeled by:

$$
\boldsymbol{\tau}_{\mathrm{iSTT}, i}=\delta_{i 1} \eta\left(\theta_{i \mathrm{p}}\right) \frac{\gamma}{M_{\mathrm{s}} \Delta} \frac{\hbar}{2} \frac{j}{e} \hat{\mathbf{m}}_{1} \times \hat{\mathbf{m}}_{1} \times \hat{\mathbf{p}} \text {. }
$$

Like the interlayer exchange interaction, the interfacial STT is a boundary effect, directly influencing only the first spin in the chain $(i=1)$, hence the appearance of the Kronecker delta function $\delta_{i 1}$ in the expression above. Constants $\hbar(\mathrm{erg} \cdot \mathrm{s})$ and $e(\mathrm{C})$ are the reduced Plank's constant and the elementary electric charge. The electric current density is given by $j\left(\mathrm{~A} / \mathrm{cm}^{2}\right)$, while $\hat{\mathbf{p}}$ denotes the unit direction vector of the magnetization of the polarizer. The angular dependence of the STT efficiency is specified by

$$
\eta\left(\theta_{\mathrm{lp}}\right)=\frac{q_{\mathrm{p}}}{A+B \cos \theta_{\mathrm{lp}}}+\frac{q_{\mathrm{p}}}{A-B \cos \theta_{\mathrm{lp}}} .
$$

Here, ${ }^{q_{\mathrm{n}}},{ }^{q_{\mathrm{n}}}, A$, and $B$ are device-dependent constants [ref., Xiao et al., 2005] and $\cos \theta_{1 \mathrm{p}}=\hat{\mathbf{m}}_{1} \cdot \hat{\mathbf{p}}$. In words, $\theta_{1 \mathrm{p}}$ is the angle between the directions of the first spin in the chain and the adjacent polarizer magnetization.

The bulk STT contribution to the dynamics of the $1 \mathrm{D}$ chain of spins, appearing in Eq. (1), is expressed as

$$
\boldsymbol{\tau}_{\mathrm{bSTT}_{i}}=u \hat{\mathbf{m}}_{i} \times \hat{\mathbf{m}}_{i} \times\left(\frac{\zeta_{i} \hat{\mathbf{m}}_{i+1}-\zeta_{i} \hat{\mathbf{m}}_{i-1}}{2 \Delta}\right)+\beta u \hat{\mathbf{m}}_{i} \times\left(\frac{\zeta_{i} \hat{\mathbf{m}}_{i+1}-\zeta_{i} \hat{\mathbf{m}}_{i-1}}{2 \Delta}\right),
$$


where $\zeta_{i}=2\left(1-\delta_{i+1, N+1}\right) /\left(2-\delta_{i-1,0}\right)$ and $\bar{\zeta}_{i}=2\left(1-\delta_{i-1,0}\right) /\left(2-\delta_{i+1, N+1}\right)$ are indicator switch functions, converting the finite-difference expressions in the parentheses above to forward differences for $i=1$, central differences for $i \in[2, N-1]$, and backward differences for $i=N$. The velocity parameter $u(\mathrm{~cm} / \mathrm{s})$, appearing in Eq. (A6), is given by

$$
u=\frac{1}{M_{\mathrm{s}}} \frac{g \mu_{\mathrm{B}}}{2 e} P j
$$

with $g$ (unitless) and $\mu_{\mathrm{B}}$ (emu) being the electron g-factor (taken as negative) and the Bohr magneton. The nonadiabaticity factor, accounting for the mistracking between carrier spins and the nonuniform magnetic moment of the free layer, is denoted by $\beta_{9}$.

The net free-layer magnetic moment $\boldsymbol{\mu}_{\text {net }}(\mathrm{emu})$ is obtained from

$$
\boldsymbol{\mu}_{\mathrm{net}}=\frac{1}{N} M_{\mathrm{s}} V \sum_{i=1}^{N} \hat{\mathbf{m}}_{i}
$$

with $V=w^{2} l$ being the volume of the free layer. The net torque $\mathbf{T}_{\text {net }}(\mathrm{emu} / \mathrm{s})$ acting on the net magnetic moment $\boldsymbol{\mu}_{\text {net }}$, in the absence of magnetostatic and magnetocrystalline anisotropy fields is

$$
\mathbf{T}_{\text {net }}=\mathbf{T}_{\mathrm{iSTT}}+\mathbf{T}_{\text {iex }}+\mathbf{T}_{\mathrm{damp}},
$$

where $\mathbf{T}_{\text {iSTT }}$ is the net interfacial STT, $\mathbf{T}_{\text {iex }}$ is the net interlayer exchange torque, and $\mathbf{T}_{\text {damp }}$ is the net damping torque. According to the principle of action-reaction, the net torque due to the bulk exchange interaction is zero, i.e., the chain of coupled spins cannot exert a net torque upon itself. Consequently, $\mathbf{T}_{\text {ex }}$ ( $=\mathbf{0}$ ) does not appear in Eq. (A9). By simplifying the vector product in Eq. (A4) for the interfacial STT, we obtain a simplified expression for $\mathbf{T}_{\mathrm{iSTT}}$ :

$$
\mathbf{T}_{\mathrm{iSTT}}=\frac{1}{N} M_{\mathrm{s}} V \boldsymbol{\tau}_{\mathrm{iSTT}, 1}=\gamma \frac{\hbar}{2} \frac{I}{e} \hat{\mathbf{m}}_{1} \times \hat{\mathbf{m}}_{1} \times \hat{\mathbf{p}}=\gamma \frac{\hbar}{2} \frac{I}{e} \sin \theta_{1} \hat{\boldsymbol{\theta}}_{1}
$$

where $\hat{\mathbf{m}}_{1} \times \hat{\mathbf{m}}_{1} \times \hat{\mathbf{p}}=\sin \theta_{1 \mathrm{p}}=\sin \theta_{1}$. In the equation above, $\hat{\boldsymbol{\theta}}_{1}=\left(\begin{array}{lll}\cos \theta_{1} \cos \phi_{1} & \cos \theta_{1} \sin \phi_{1} & -\sin \theta_{1}\end{array}\right)$ is the unit vector in the polar direction, and $\hat{\phi}_{1}=\left(\begin{array}{lll}-\sin \phi_{1} & \cos \phi_{1} & 0\end{array}\right)$ is the unit vector in the azimuthal direction, given here in Cartesian form, with $\theta_{1}$ and $\phi_{1}$ representing the polar and azimuthal directions of the first spin in the chain with respect to the coordinate system shown in Fig. 1d.

The interlayer exchange torque exerted on the free-layer magnetic moment, according to Eq. (A2), is

$$
\mathbf{T}_{\mathrm{iex}}=\frac{1}{N} M_{\mathrm{s}} V \boldsymbol{\tau}_{\mathrm{iex}, N}=-\gamma \frac{1}{N} M_{\mathrm{s}} V \hat{\mathbf{m}}_{N} \times \mathbf{H}_{\mathrm{iex}, N}=-\gamma J_{\mathrm{ex}} S \hat{\mathbf{m}}_{N} \times \hat{\mathbf{q}}=q_{z} \gamma J_{\mathrm{ex}} S \sin \theta_{N} \hat{\boldsymbol{\phi}}_{N}
$$


where $S=w^{2}$ is the area of the cross section of the free layer, $\hat{\mathbf{m}}_{N} \times \hat{\mathbf{q}}=-q_{z} \sin \theta_{N \mathrm{q}} \hat{\boldsymbol{\phi}}_{N}=-q_{z} \sin \theta_{N} \hat{\boldsymbol{\phi}}_{N}$, and a vertical magnetization of the pinned layer is assumed, i.e., $\mathbf{q}=\left(\begin{array}{lll}0 & 0 & q_{z}\end{array}\right)$. In cases 0 and 1 (Fig. 1a,b), we have that $q_{z}=-1$, while in case 2 (Fig. 1c), we have $q_{z}=1$.

Finally, the net damping torque on the free layer, according to the second term on the right-hand side of Eq. (1), is

$$
\mathbf{T}_{\mathrm{damp}}=\frac{1}{N} M_{\mathrm{s}} V \boldsymbol{\tau}_{\mathrm{damp}}=\frac{1}{N} M_{\mathrm{s}} V \alpha \sum_{i=1}^{N} \hat{\mathbf{m}}_{i} \times \frac{d \hat{\mathbf{m}}_{i}}{d t}=-2 \pi f \alpha M_{\mathrm{s}} V \frac{1}{N} \sum_{i=1}^{N} \sin \theta_{i} \hat{\boldsymbol{\theta}}_{i}
$$

In expressing Eq. (A12), we have made use of the knowledge that, due to symmetry, and as observed in simulations, the precession of the chain of spins is occurring at steady-state about the vertical magnetization direction of the pinned layer. Therefore,

$$
\frac{d \hat{\mathbf{m}}_{i}}{d t}=2 \pi f \sin \theta_{i} \hat{\phi}_{i}
$$

where $f>0$ for precession in the ${ }^{+\hat{\boldsymbol{\phi}}_{i}}$ direction (counter-clockwise (CCW) when viewed from the top), and $f<0$ for precession the $-\hat{\phi}_{i}$ (CW) direction. From Eq. (A13) it follows that $\hat{\mathbf{m}}_{i} \times d \hat{\mathbf{m}}_{i} / d t=-2 \pi f \sin \theta_{i} \hat{\boldsymbol{\theta}}_{i}$, as expressed in Eq. (A12). The occurrence of the oscillation frequency $f$ in Eq. (A12) for $\mathbf{T}_{\text {damp }}$ is expected, as damping is of the viscous type.

\section{References}

1. J. Torrejon, M. Riou, F. A. Araujo, S. Tsunegi, G. Khalsa, D. Querlioz, P. Bortolotti, V. Cros, K. Yakushiji, A. Fukushima, H. Kubota, S. Y. Uasa, M. D. Stiles and J. Grollier, Nature 547 (7664), 428-431 (2017).

2. M. Romera, P. Talatchian, S. Tsunegi, F. A. Araujo, V. Cros, P. Bortolotti, J. Trastoy, K. Yakushiji, A. Fukushima, H. Kubota, S. Yuasa, M. Ernoult, D. Vodenicarevic, T. Hirtzlin, N. Locatelli, D. Querlioz and J. Grollier, Nature 563 (7730), 230-234 (2018).

3. S. E. Russek, W. H. Rippard, T. Cecil and R. Heindl, in Functional Nanomaterials, Handbook of Nanophysics, edited by K. D. Sattler (CRC Press, Boca Raton, 2010).

4. Z. Zeng, G. Finocchio and H. Jiang, Nanoscale 5 (6), 2219-2231 (2013).

5. J. A. Katine and E. E. Fullerton, J. Magn. Magn. Mater. 320 (7), 1217-1226 (2008).

6. T. S. Chen, R. K. Dumas, A. Eklund, P. K. Muduli, A. Houshang, A. A. Awad, P. Durrenfeld, B. G. Malm, A. Rusu and J. Akerman, Proc. IEEE 104 (10), 1919-1945 (2016).

7. L. Berger, Phys. Rev. B 54 (13), 9353-9358 (1996).

8. J. C. Slonczewski, J. Magn. Magn. Mater. 159 (1-2), L1-L7 (1996).

9. S. Zhang and Z. Li, Phys. Rev. Lett. 93 (12) (2004).

10. A. Thiaville, Y. Nakatani, J. Miltat and Y. Suzuki, Europhysics Letters 69 (6), 990-996 (2005). 
11. D. C. Ralph and M. D. Stiles, J. Magn. Magn. Mater. 320 (7), 1190-1216 (2008).

12. J. E. Hirsch, Phys. Rev. Lett. 83 (9), 1834-1837 (1999).

13. J. Sinova, S. O. Valenzuela, J. Wunderlich, C. H. Back and T. Jungwirth, Reviews of Modern Physics 87 (4), 1213-1259 (2015).

14. A. Manchon, J. Zelezny, I. M. Miron, T. Jungwirth, J. Sinova, A. Thiaville, K. Garello and P. Gambardella, Reviews of Modern Physics 91 (3) (2019).

15. F. Hellman, A. Hoffmann, Y. Tserkovnyak, G. S. D. Beach, E. E. Fullerton, C. Leighton, A. H. MacDonald, D. C. Ralph, D. A. Arena, H. A. Durr, P. Fischer, J. Grollier, J. P. Heremans, T. Jungwirth, A. V. Kimel, B. Koopmans, I. N. Krivorotov, S. J. May, A. K. Petford-Long, J. M. Rondinelli, N. Samarth, I. K. Schuller, A. N. Slavin, M. D. Stiles, O. Tchernyshyov, A. Thiaville and B. L. Zink, Reviews of Modern Physics 89 (2) (2017).

16. M. N. Baibich, J. M. Broto, A. Fert, F. N. Vandau, F. Petroff, P. Eitenne, G. Creuzet, A. Friederich and J. Chazelas, Phys. Rev. Lett. 61 (21), 2472-2475 (1988).

17. G. Binasch, P. Grunberg, F. Saurenbach and W. Zinn, Phys. Rev. B 39 (7), 4828-4830 (1989).

18. M. Julliere, Physics Letters A 54 (3), 225-226 (1975).

19. M. Tsoi, A. G. M. Jansen, J. Bass, W. C. Chiang, V. Tsoi and P. Wyder, Nature 406 (6791), 46-48 (2000).

20. S. I. Kiselev, J. C. Sankey, I. N. Krivorotov, N. C. Emley, R. J. Schoelkopf, R. A. Buhrman and D. C. Ralph, Nature 425 (6956), 380-383 (2003).

21. W. H. Rippard, M. R. Pufall, S. Kaka, S. E. Russek and T. J. Silva, Phys. Rev. Lett. 92 (2) (2004).

22. S. Kaka, M. R. Pufall, W. H. Rippard, T. J. Silva, S. E. Russek and J. A. Katine, Nature 437 (7057), 389-392 (2005).

23. A. M. Deac, A. Fukushima, H. Kubota, H. Maehara, Y. Suzuki, S. Yuasa, Y. Nagamine, K. Tsunekawa, D. D. Djayaprawira and N. Watanabe, Nat. Phys. 4 (10), 803-809 (2008).

24. A. Slavin and V. Tiberkevich, Ieee Transactions on Magnetics 45 (4), 1875-1918 (2009).

25. Z. M. Zeng, G. Finocchio, B. S. Zhang, P. K. Amiri, J. A. Katine, I. N. Krivorotov, Y. M. Huai, J. Langer, B. Azzerboni, K. L. Wang and H. W. Jiang, Sci Rep 3 (2013).

26. R. H. Liu, W. L. Lim and S. Urazhdin, Phys. Rev. Lett. 110 (14) (2013).

27. Z. Duan, A. Smith, L. Yang, B. Youngblood, J. Lindner, V. E. Demidov, S. O. Demokritov and I. N. Krivorotov, Nature Communications 5 (2014).

28. Y. C. Lau, D. Betto, K. Rode, J. M. D. Coey and P. Stamenov, Nature Nanotechnology 11 (9), 758-762 (2016).

29. R. Cheng, D. Xiao and A. Brataas, Phys. Rev. Lett. 116 (20) (2016).

30. F. Garcia-Sanchez, J. Sampaio, N. Reyren, V. Cros and J. V. Kim, New J. Phys. 18 (2016).

31. C. Safranski, I. Barsukov, H. K. Lee, T. Schneider, A. A. Jara, A. Smith, H. Chang, K. Lenz, J. Lindner, Y. Tserkovnyak, M. Wu and I. N. Krivorotov, Nature Communications 8 (2017).

32. C. D. Jin, J. B. Wang, W. W. Wang, C. K. Song, J. S. Wang, H. Y. Xia and Q. F. Liu, Physical Review Applied 9 (4) (2018).

33. F. B. Mancoff, N. D. Rizzo, B. N. Engel and S. Tehrani, Nature 437 (7057), 393-395 (2005).

34. T. J. Silva and W. H. Rippard, J. Magn. Magn. Mater. 320 (7), 1260-1271 (2008).

35. M. Manfrini, T. Devolder, J. V. Kim, P. Crozat, N. Zerounian, C. Chappert, W. Van Roy, L. Lagae, G. Hrkac and T. Schrefl, Appl. Phys. Lett. 95 (19) (2009).

36. S. Bonetti, P. Muduli, F. Mancoff and J. Akerman, Appl. Phys. Lett. 94 (10) (2009). 
37. R. K. Dumas, S. R. Sani, S. M. Mohseni, E. Iacocca, Y. Pogoryelov, P. K. Muduli, S. Chung, P. Durrenfeld and J. Akerman, Ieee Transactions on Magnetics 50 (6) (2014).

38. V. S. Pribiag, I. N. Krivorotov, G. D. Fuchs, P. M. Braganca, O. Ozatay, J. C. Sankey, D. C. Ralph and R. A. Buhrman, Nat. Phys. 3 (7), 498-503 (2007).

39. D. Houssameddine, U. Ebels, B. Delaet, B. Rodmacq, I. Firastrau, F. Ponthenier, M. Brunet, C. Thirion, J. P. Michel, L. Prejbeanu-Buda, M. C. Cyrille, O. Redon and B. Dieny, Nature Materials 6 (6), 447-453 (2007).

40. I. Firastrau, D. Gusakova, D. Houssameddine, U. Ebels, M. C. Cyrille, B. Delaet, B. Dieny, O. Redon, J. C. Toussaint and L. D. Buda-Prejbeanu, Phys. Rev. B 78 (2) (2008).

41. H. Zhang, W. W. Lin, S. Mangin, Z. Z. Zhang and Y. W. Liu, Appl. Phys. Lett. 102 (1) (2013).

42. Y. Zhou and J. Akerman, Appl. Phys. Lett. 94 (11) (2009).

43. P. K. Muduli, O. G. Heinonen and J. Akerman, Journal of Applied Physics 110 (7) (2011).

44. L. Q. Liu, C. F. Pai, D. C. Ralph and R. A. Buhrman, Phys. Rev. Lett. 109 (18) (2012).

45. P. K. Muduli, O. G. Heinonen and J. Akerman, Phys. Rev. Lett. 108 (20) (2012).

46. H. Kubota, K. Yakushiji, A. Fukushima, S. Tamaru, M. Konoto, T. Nozaki, S. Ishibashi, T. Saruya, S. Yuasa, T. Taniguchi, H. Arai and H. Imamura, Applied Physics Express 6 (10) (2013).

47. T. Ono and Y. Nakatani, Applied Physics Express 1 (6) (2008).

48. L. Yang, R. Verba, V. Tiberkevich, T. Schneider, A. Smith, Z. Duan, B. Youngblood, K. Lenz, J. Lindner, A. N. Slavin and I. N. Krivorotov, Sci Rep 5 (2015)

49. J. He and S. Zhang, Appl. Phys. Lett. 90 (14) (2007).

50. M. Franchin, T. Fischbacher, G. Bordignon, P. de Groot and H. Fangohr, Phys. Rev. B 78 (5) (2008).

51. A. Bisig, L. Heyne, O. Boulle and M. Klaui, Appl. Phys. Lett. 95 (16) (2009).

52. K. Matsushita, J. Sato and H. Imamura, Journal of the Physical Society of Japan 78 (9) (2009).

53. J. H. Franken, R. Lavrijsen, J. T. Kohlhepp, H. J. M. Swagten and B. Koopmans, Appl. Phys. Lett. 98 (10) (2011).

54. M. Doi, H. Endo, K. Shirafuji, S. Kawasaki, M. Sahashi, H. N. Fuke, H. Iwasaki and H. Imamura, Journal of Physics DApplied Physics 44 (9) (2011).

55. S. Sharma, B. Muralidharan and A. Tulapurkar, Sci Rep 5 (2015).

56. B. Van de Wiele, S. J. Hamalainen, P. Balaz, F. Montoncello and S. van Dijken, Sci Rep 6 (2016).

57. M. Voto, L. Lopez-Diaz and E. Martinez, Sci Rep 7 (2017).

58. B. Kruger, A. Drews, M. Bolte, U. Merkt, D. Pfannkuche and G. Meier, Phys. Rev. B 76 (22) (2007)

59. Q. Mistral, M. van Kampen, G. Hrkac, J. V. Kim, T. Devolder, P. Crozat, C. Chappert, L. Lagae and T. Schrefl, Phys. Rev. Lett. 100 (25) (2008).

60. A. Dussaux, B. Georges, J. Grollier, V. Cros, A. V. Khvalkovskiy, A. Fukushima, M. Konoto, H. Kubota, K. Yakushiji, S. Yuasa, K. A. Zvezdin, K. Ando and A. Fert, Nature Communications 1 (2010).

61. S. F. Zhang, J. B. Wang, Q. Zheng, Q. Y. Zhu, X. Y. Liu, S. J. Chen, C. D. Jin, Q. F. Liu, C. L. Jia and D. S. Xue, New J. Phys. 17 (2015).

62. R. H. Liu, W. L. Lim and S. Urazhdin, Phys. Rev. Lett. 114 (13) (2015).

63. C. P. Chui and Y. Zhou, AIP Adv. 5 (9) (2015).

64. G. Finocchio, F. Buttner, R. Tomasello, M. Carpentieri and M. Klaui, Journal of Physics D-Applied Physics 49 (42) (2016). 
65. A. Giordano, R. Verba, R. Zivieri, A. Laudani, V. Puliafito, G. Gubbiotti, R. Tomasello, G. Siracusano, B. Azzerboni, M. Carpentieri, A. Slavin and G. Finocchio, Sci Rep 6 (2016).

66. N. Perrissin, S. Lequeux, N. Strelkov, A. Chavent, L. Vila, L. D. Buda-Prejbeanu, S. Auffret, R. C. Sousa, I. L. Prejbeanu and B. Dieny, Nanoscale 10 (25), 12187-12195 (2018)

67. K. Watanabe, B. Jinnai, S. Fukami, H. Sato and H. Ohno, Nature Communications 9 (2018)

68. N. Perrissin, G. Gregoire, S. Lequeux, L. Tillie, N. Strelkov, S. Auffret, L. D. Buda-Prejbeanu, R. C. Sousa, L. B. Dieny and I. L. Prejbeanu, Journal of Physics D-Applied Physics 52 (23) (2019).

69. L. Q. Liu, T. Moriyama, D. C. Ralph and R. A. Buhrman, Phys. Rev. Lett. 106 (3) (2011)

70. K. Garello, I. M. Miron, C. O. Avci, F. Freimuth, Y. Mokrousov, S. Blugel, S. Auffret, O. Boulle, G. Gaudin and P. Gambardella, Nature Nanotechnology 8 (8), 587-593 (2013).

71. S. Woo, M. Mann, A. J. Tan, L. Caretta and G. S. D. Beach, Appl. Phys. Lett. 105 (21) (2014).

72. X. Fan, H. Celik, J. Wu, C. Y. Ni, K. J. Lee, V. O. Lorenz and J. Q. Xiao, Nature Communications 5 (2014).

73. K. Garello, C. O. Avci, I. M. Miron, M. Baumgartner, A. Ghosh, S. Auffret, O. Boulle, G. Gaudin and P. Gambardella, Appl. Phys. Lett. 105 (21) (2014).

74. S. Fukami, T. Anekawa, C. Zhang and H. Ohno, Nature Nanotechnology 11 (7), 621-625 (2016).

75. S. Fukami, C. L. Zhang, S. DuttaGupta, A. Kurenkov and H. Ohno, Nature Materials 15 (5), 535-541 (2016).

76. L. Berger, Journal of Applied Physics 55 (6), 1954-1956 (1984)

77. G. Tatara, T. Takayama, H. Kohno, J. Shibata, Y. Nakatani and H. Fukuyama, Journal of the Physical Society of Japan 75 (6) (2006).

78. C. Burrowes, A. P. Mihai, D. Ravelosona, J. V. Kim, C. Chappert, L. Vila, A. Marty, Y. Samson, F. Garcia-Sanchez, L. D. Buda-Prejbeanu, I. Tudosa, E. E. Fullerton and J. P. Attane, Nat. Phys. 6 (1), 17-21 (2010).

79. A. V. Khvalkovskiy, J. Grollier, A. Dussaux, K. A. Zvezdin and V. Cros, Phys. Rev. B 80 (14) (2009)

80. J. V. Kim, in Solid State Physics, Vol 63, edited by R. E. Camley and R. L. Stamps (2012), Vol. 63, pp. 217-294.

81. C. Fowley, V. Sluka, K. Bernert, J. Lindner, J. Fassbender, W. H. Rippard, M. R. Pufall, S. E. Russek and A. M. Deac, Applied Physics Express 7 (4) (2014).

82. R. C. O'Handley, Modern Magnetic Materials: Principles and Applications. (John Wiley \& Sons, New York, 1999).

83. K. M. D. Hals and A. Brataas, Phys. Rev. B 87 (17) (2013).

84. M. Bode, M. Heide, K. von Bergmann, P. Ferriani, S. Heinze, G. Bihlmayer, A. Kubetzka, O. Pietzsch, S. Blugel and R. Wiesendanger, Nature 447 (7141), 190-193 (2007).

85. S. Emori, U. Bauer, S. M. Ahn, E. Martinez and G. S. D. Beach, Nature Materials 12 (7), 611-616 (2013).

86. Y. Zhou, C. L. Zha, S. Bonetti, J. Persson and J. Akerman, Journal of Applied Physics 105 (7) (2009).

87. R. Law, E. L. Tan, R. Sbiaa, T. Liew and T. C. Chong, Appl. Phys. Lett. 94 (6) (2009).

88. R. Sbiaa, S. Y. H. Lua, R. Law, H. Meng, R. Lye and H. K. Tan, Journal of Applied Physics 109 (7) (2011).

89. S. M. Mohseni, S. R. Sani, J. Persson, T. N. Nguyen, S. Chung, Y. Pogoryelov and J. Akerman, Physica Status Solidi-Rapid Research Letters 5 (12), 432-434 (2011).

90. P. M. Braganca, J. A. Katine, N. C. Emley, D. Mauri, J. R. Childress, P. M. Rice, E. Delenia, D. C. Ralph and R. A. Buhrman, Ieee Transactions on Nanotechnology 8 (2), 190-195 (2009).

91. S. W. Lee and K. J. Lee, Proc. IEEE 104 (10), 1831-1843 (2016). 


\title{
Current driven transverse domain wall oscillations in perpendicular spin valve structure
}

\author{
M. V. Lubarda ${ }^{1-3}$, M. Kuteifan ${ }^{2,3}$, C.-H. Lambert ${ }^{3}$, V. Lomakin², E. E. Fullerton², S. Mangin ${ }^{3}$
}

${ }^{1}$ Faculty of Polytechnics, University of Donja Gorica, Oktoih 1, 81000 Podgorica, Montenegro

${ }^{2}$ Center of Memory and Recording Research, University of California, San Diego, California 92093-0401, USA

${ }^{3}$ Institut Jean Lamour, UMR CNRS 7198, Université de Lorraine, 54506 Vandoeuvre lès Nancy, France

\section{Supplementary Material}

In the following supplementary sections, we elaborate further on the interplay between different interactions and illustrate the effects of the damping constant, spin polarization efficiency, free-layer length, and other material and structural properties on the oscillation frequency of the studied STO in the three cases of interest (Fig. 1a-c).

\section{Case 0: additional characteristics}

To gain further insight into the response of the STO in case 0 , we consider the DW position, $z_{\mathrm{DW}}$, as a function of the applied current, and examine the influence of material and structural parameters on STO frequency.

Figure S1 shows that the DW in the free layer is located closest to the pinned layer when a particular value of current is reached resulting in maximum net torque, $T_{\text {net }}$ (Fig. 5a), and near-maximum absolute frequency, ${ }^{|f|}$ (Fig. Erreur ! Source du renvoi introuvable.a). As can be expected, when a DW is closest to the pinned layer, the angle between the topmost spin in the free-layer chain and the pinned-layer magnetic moment reaches a maximum value, resulting in a large interlayer exchange torque and, consequently, more rapid oscillations.

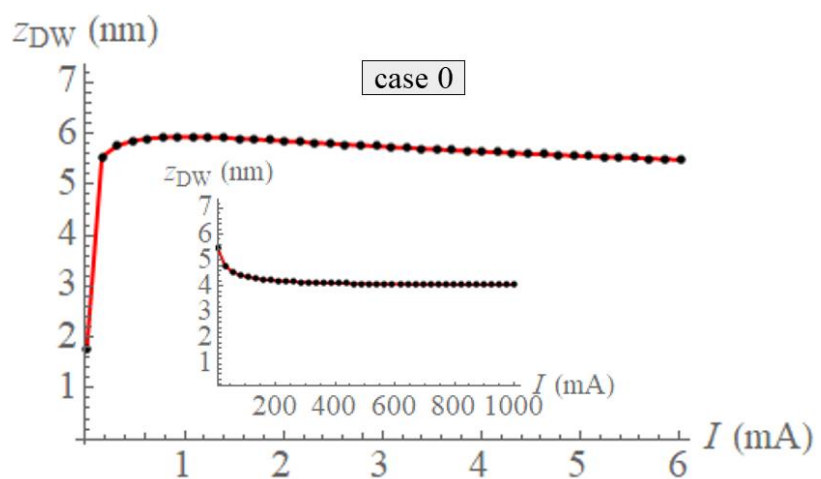

Figure S1: Domain wall position as a function of applied current in case 0 . As the current is initially increased the DW introduced into the free layer by the action of the interfacial STT climbs increasingly toward the freelayer/pinned-layer interface. Maximum frequency of magnetization oscillations is reached at a current value for which the DW is approximately closest to this interface (Fig. Erreur ! Source du renvoi introuvable.a,biv), since the interlayer exchange torque which generates precession of the free layer moment is then at its 
highest value. For current increases beyond the maximum-frequency mark, the DW moves gradually downward, ultimately settling near the midpoint of the free layer in the limit $I \rightarrow \infty$ (figure inset).

The initial climb of the DW toward the pinned layer with increasing current, and its subsequent retraction upon further current increase, as observed in Figs. Erreur ! Source du renvoi introuvable.d and S1, is not due directly to the effect of the interfacial STT, since in case 0 the STT acts only interfacially, and not throughout the bulk of the free layer. The observed DW-displacement behavior is instead related to the aforementioned coupling between the polar and azimuthal degrees of freedom of the spin chain. It is due to this coupling and the problem-specific boundary conditions that the twist of the spin chain continuously increases with increasing current, even when the oscillation absolute frequency $|f|$ becomes negatively correlated with $I$, in the high current regime.

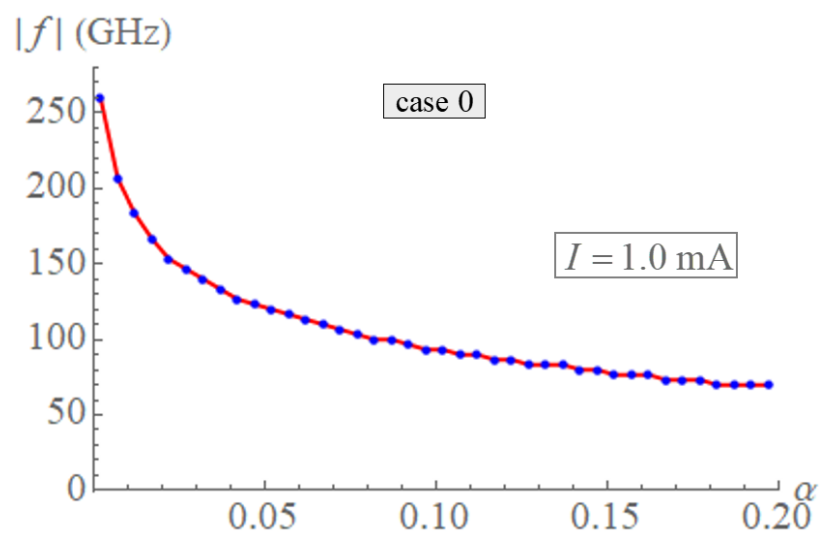

Figure S2: Frequency of precession as a function of free-layer damping constant for a given applied electric current in case 0 .

Figure $\mathrm{S} 2$ shows that for a greater free-layer damping constant $\alpha$ the frequency of oscillations for a given current value is reduced. This is expected, as the damping torque, $\boldsymbol{\tau}_{\text {damp, } i}$, acting upon each spin along the $\hat{\boldsymbol{\theta}}_{i}$

${ }^{i}$ direction, tends to bring about greater uniformity of fanning of the spin chain (Fig. 4a(i)). We, therefore, expect more even fanning for a larger damping constant $\alpha$ at a given applied current. Since nonuniform fanning of the spins gives rise to the azimuthal component of the bulk exchange torque, ${ }^{\boldsymbol{e} x, i}$, which generates precession of each interior spin, we conclude that the precessional frequency is negatively correlated with $\alpha$, as confirmed by simulations (Fig. S2).

In Fig. S3, we see that reducing the free-layer saturation magnetization, $M_{\mathrm{s}}$, results in a higher rate of magnetization oscillations. More exactly, for a given current we observe that the oscillation frequency is inversely proportional to the saturation magnetization, i.e., $|f| \propto 1 / M_{\mathrm{s}}$. This dependence is expected considering that the precessional inertia of the free layer is equal to the in-plane component of its magnetic moment, $\quad \mu_{\square}=M_{\mathrm{s}} \frac{1}{N} \sum_{i=1} m_{i}$, and $2 \pi f=T_{\text {net }} / \mu$. Furthermore, since the DW length, $l_{\mathrm{DW}} \propto \frac{1}{N} \sum_{i=1} m_{i}$, 
factors into the precessional inertia ${ }^{\mu}$, we conclude that for a given net torque $T_{\text {net }}$, the precession is faster for a more confined DW.

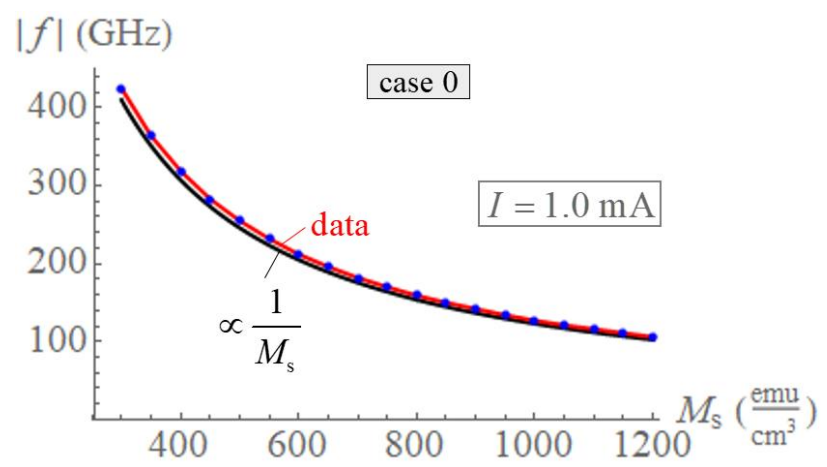

Figure S3: Frequency of precession as a function of free-layer saturation magnetization for a given applied electric current in case 0 , showing a reciprocal relationship.

As the images of spin configurations for different current values in Fig. 2d show, under the action of the spin-polarized current, a DW develops in the free layer. The precession of the DW magnetic moment is generated by the interlayer exchange torque, $\mathbf{T}_{\mathrm{iex}}=-\gamma J_{\mathrm{ex}} S \sin \theta \hat{\boldsymbol{\phi}}$, as Fig. 5a shows. The maximum interlayer exchange torque that the pinned layer can exert on the free-layer magnetic moment, therefore, is $\max \left(\left|\mathbf{T}_{\text {iex }}\right|\right)=\gamma J_{\text {ex }} S$. Since longer free layers generally have more magnetic moment owing to an increased volume, it is expected that for a given applied current oscillations are slower in longer layers. Figure $\mathrm{S} 4$ illustrates the highly sensitive oscillation dependence on free-layer length, indicating that $|f| \propto 1 / l^{2}$.

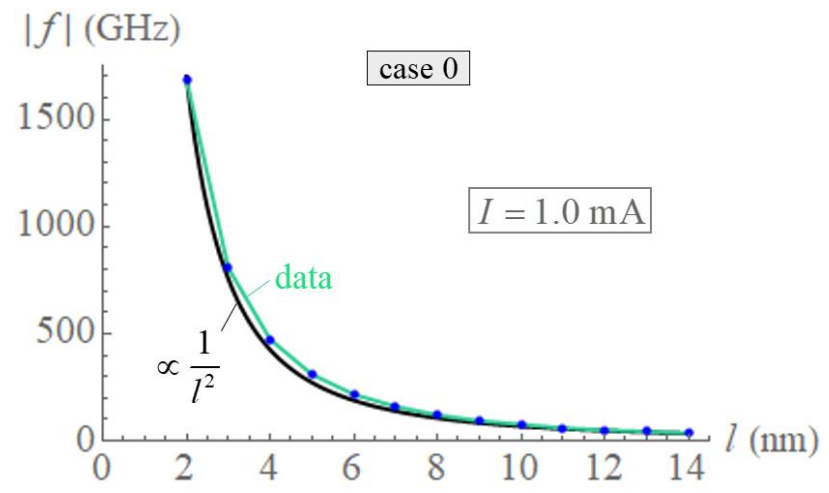

Figure S4: Frequency of precession as a function of free-layer length for a given applied electric current in case 0 . 
Based on the torque analysis for an arbitrary interior spin summarized in Fig. 4a(i) and Eqs. (1) and (A1), we can conclude that the frequency is linearly proportional to the free-layer bulk exchange stiffness, $A_{\mathrm{ex}}$. If, for example, during steady-state precession $A_{\mathrm{ex}}$ would suddenly double, the azimuthal component of the bulk exchange torque, ${ }^{\boldsymbol{\tau}_{\mathrm{ex}, i}}$, would consequently double, generating twice as fast precession. Due to the double increase in the rate of precession about the vertical axis, the damping torque, $\boldsymbol{\tau}_{\text {damp }, i}=\tau_{\text {damp }, i} \hat{\boldsymbol{\theta}}_{i}$, would necessarily double as well. Still, $\boldsymbol{\tau}_{\mathrm{damp}, i}$ is perfectly compensated by the polar component of the bulk exchange torque, ${ }^{\theta}{ }_{\mathrm{ex}, i}$, which, being proportional to $A_{\mathrm{ex}}$, would also double. Hence, the spin chain does not change its profile, only its rate of precession would have doubled. Figure S5 shows the expected linear dependence of oscillation frequency on $A_{\mathrm{ex}}$ as obtained by simulations.

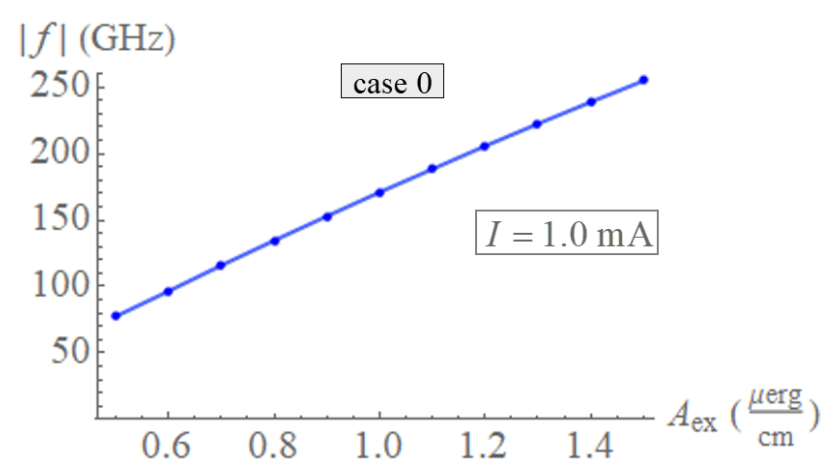

Figure S5: Frequency of precession as a function of free-layer exchange stiffness for a given applied electric current in case 0 , showing a linear relationship.

Next, we consider the effect of perpendicular magnetocrystalline anisotropy on the STO characteristics. $\underset{\boldsymbol{\tau}_{\text {anis }, i}=-\gamma \hat{\mathbf{m}}_{i}}{\text { According }} \times \mathbf{H}_{\text {anis }, i} \stackrel{\text { to }}{=} \gamma \frac{2 K(1)}{M} \sin \hat{\theta}_{i} \cos \theta_{i} \hat{\phi}_{i}$ A3), the magnetocrystalline anisotropy torque, , acts on each spin in the azimuthal direction $\phi_{i}$, with a strength proportional to $\sin \theta_{i} \cos \theta_{i} \propto \sin 2 \theta_{i}$, and an orientation that depends on the sign of the vertical projection of the spin, $m_{i}^{z}=\cos \theta_{i}$. For a current value (e.g., $\left.I=1 \mathrm{~mA}\right)$ for which a sufficient majority portion of the free-layer spins are oriented in the upward direction (Fig. $2 \mathrm{~d}-i v$ ), the overall anisotropy torque is in the ${ }^{+\boldsymbol{\phi}_{i}}$ direction (since $\theta_{i}>\pi / 2$ for most spins), and thus it opposes the action of the interlayer exchange torque and decrease the rate of CW precession with increasing $K_{\mathrm{u}}$, as seen in Fig. S6. 


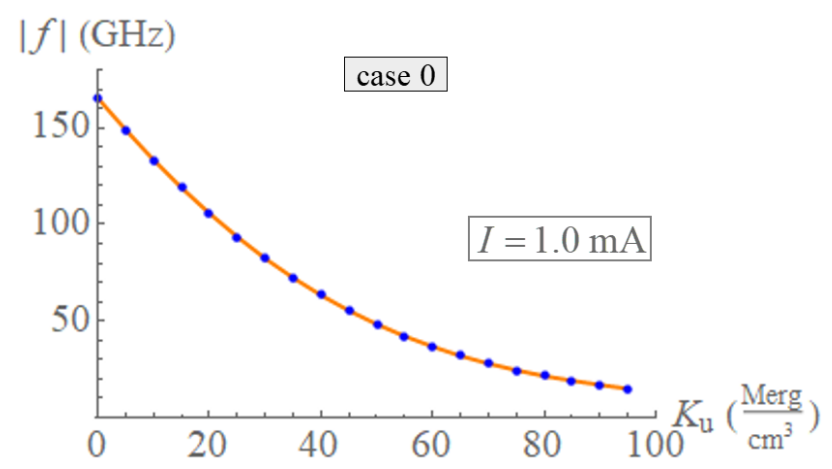

Figure S6: Frequency of precession as a function of the perpendicular-magnetocrystalline-anisotropy constant of the free layer for a given applied electric current in case 0 .

Conversely, for low currents (e.g., $I=0.01 \mathrm{~mA}$ ), when a sufficient majority of spins have a negative vertical projection (Fig. 2d-ii), the net anisotropy torque on these spins exceeds that exerted over the spins with a positive vertical projection, and the overall anisotropy torque constructively superimposes with the interlayer exchange torque, thus accelerating the precession with increasing $K_{\mathrm{u}}$. However, simulations reveal that increasing $K_{\mathrm{u}}$ also increases the threshold current required for sustained magnetization precession to such a level that the range of currents over which most spins have a negative vertical projection becomes close to negligible.

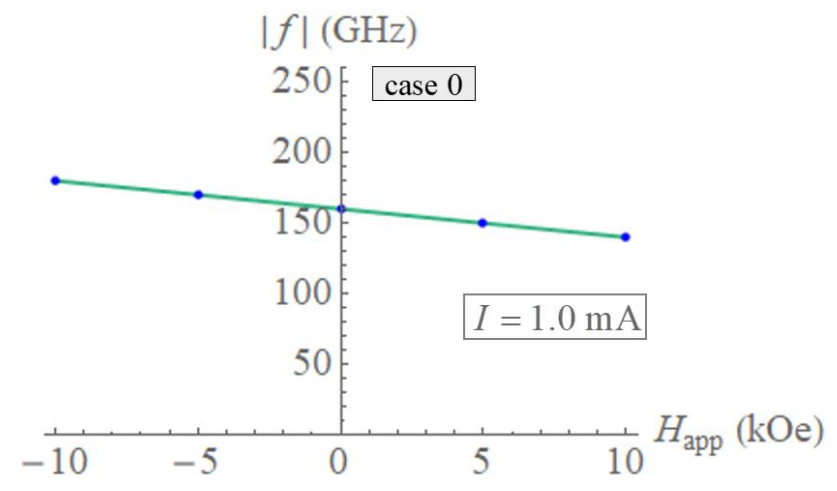

Figure S7: Frequency of precession as a function of the vertically applied magnetic field for a given applied electric current in case 0 .

While an externally applied magnetic field is not required for sustained oscillations of the considered STO, its effect on the precessional frequency is of interest, nonetheless. We limit our considerations to a vertical applied field, $\mathbf{H}_{\text {app }}=H_{\text {app }} \mathbf{z}$. Figure S7 shows that the frequency of oscillations is linearly dependent on $H_{\text {app }}$. When the field is applied in the direction of the pinned-layer magnetization ( $H_{\text {app }}<0$ ), the frequency of oscillations increases, since the interlayer exchange field and the applied field act in the same direction, 
hence the torque from the applied field assists the interlayer exchange torque in the generation of magnetization precession. On the contrary, when the external field is applied counter to the pinned layer magnetization, their respective torques compete, and the frequency of oscillations is reduced.

\section{Case 1: additional characteristics}

Figure S8 shows the inverse square dependence of frequency on the damping constant in case 1, which is in agreement with the derivations by Franchin, et al. For ultra-high-frequency oscillations, a free layer with a much-reduced damping constant would be desirable. The strong dependence of frequency on $\alpha$ suggests that variations of the damping constant within the free layer due to compositional variations, impurities, or temperature fluctuations, as may occur in actual systems in operation, would be a possible source of oscillation decoherence that would contribute to spectral-linewidth broadening.

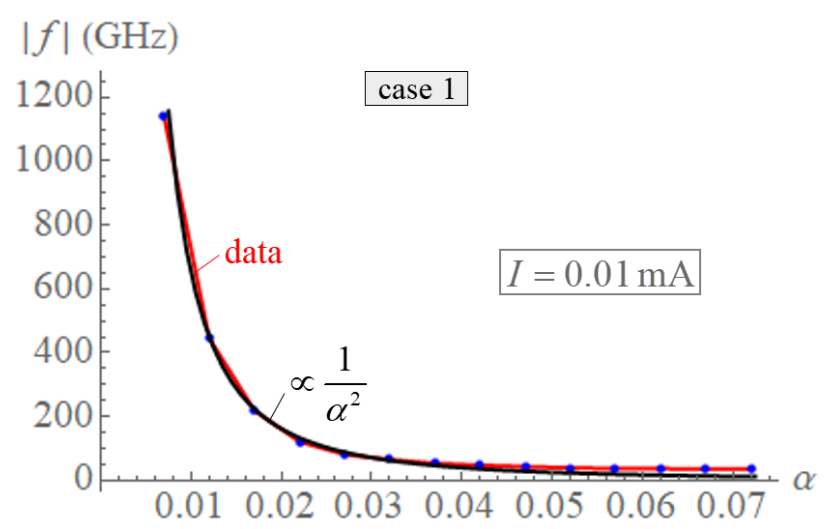

Figure S8: Frequency of precession as a function of free-layer damping constant for a given applied electric current in case 1 .

Figure S9 shows that the oscillation frequency is sensitively dependent on the free-layer thickness ${ }^{50}$. The shorter the free layer is, the more compressed against the pinned layer the DW will be, which leads to an increased precession-generating interlayer exchange torque. A shorter free layer, moreover, implies a reduced net magnetic moment, and hence more rapid oscillations for the same torque magnitude. For a given applied current, however, there exists a minimum free-layer length under which steady-state oscillations are not sustainable. For the STO described here, precessions do not persist for free-layer lengths less than $2 \mathrm{~nm}$. 


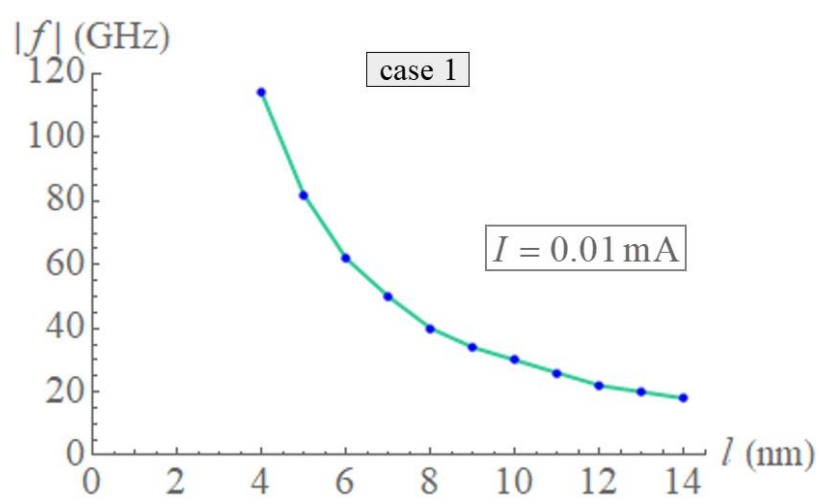

Figure S9: Frequency of precession as a function of free-layer length for a given applied electric current in case 1.

Figure $\mathrm{S} 10$ shows that the oscillation frequency in case 1 is not proportional to the free-layer exchange stiffness, $A_{\mathrm{ex}}$, as in case 0 (Fig. S5). This is because the compensation of torques at each interior spin in the two cases are different; in case 0: $\boldsymbol{\tau}_{\mathrm{ex}, i}^{\theta}+\boldsymbol{\tau}_{\mathrm{damp}, i}=\mathbf{0}$ (Fig. 4a(i)), whereas in case 1: $\boldsymbol{\tau}_{\mathrm{bSTT}, i}^{\theta}+\boldsymbol{\tau}_{\mathrm{damp}, i}=\mathbf{0}$ (Fig. 4b(i)). The frequency in case 1, as a result, has a nonlinear dependence on $A_{\text {ex }}$. As the exchange stiffness increases, the DW expands to reduce magnetization nonuniformity, hence the in-plane component of the magnetic moment (precessional inertia) increases, which contributes to the slowing down of oscillations.

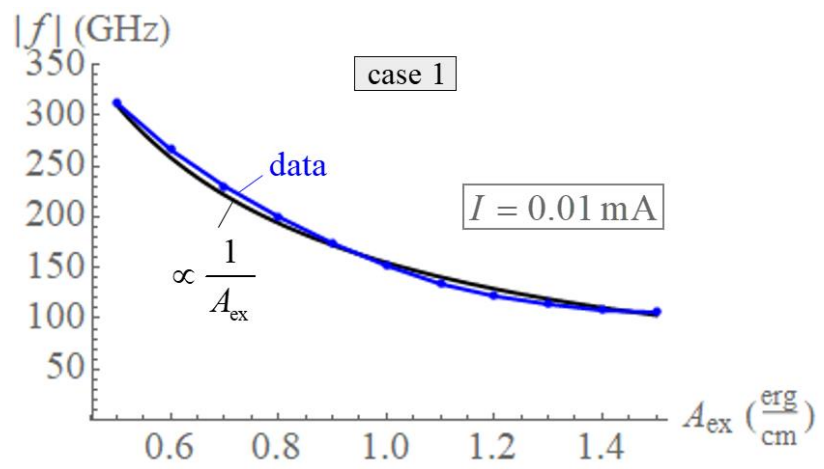

Figure S10: Frequency of precession as a function of free-layer exchange stiffness for a given applied electric current in case 1, showing a reciprocal relationship.

Simulations show that with an increase in perpendicular magnetocrystalline anisotropy of the free layer, the rate of oscillations increases (Fig. S11). The effect of the perpendicular magnetocrystalline anisotropy in case 2 is to reduce the width of the DW, whereby the precessional inertia decreases and the rate of oscillations increases 


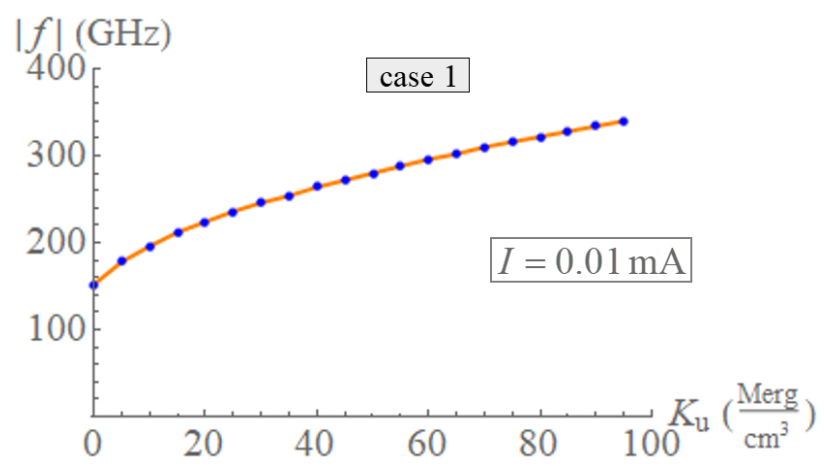

Figure S11: Frequency of precession as a function of free-layer perpendicular-magnetocrystalline-anisotropy constant for a given applied electric current in case 1.

While reducing the length and/or the exchange stiffness of the free layer, and/or increasing its perpendicular magnetocrystalline anisotropy, may result in large increases in oscillation rate, which is appealing from a high-frequency applications perspective, it may also result in increased sensitivity of oscillations to disturbances coming from thermal agitation of the free layer magnetization and electronic noise. This is because optimization for ultra-high frequency operation in case 1 implies a reduced oscillating component of the free-layer magnetic moment (narrower DW), with an implied greater susceptibility to phase decoherence from stochastic effects.

\section{Case 2: additional characteristics}

In case 2, where the vertical components of the interfacial and the bulk STT compensate each other, dissipation, interestingly, does not affect STO response appreciably, as illustrated in Fig. S12. The oscillation frequency is seen to be nearly independent of the damping constant $\alpha$. The weak dependence on $\alpha$ suggests increased coherence of oscillations, as variations in the damping constant due to compositional inhomogeneities, impurities, defects, and thermal-field fluctuations are anticipated to factor much less in spectral linewidth broadening. Such an insensitivity to damping implies that a much broader selection of ferromagnetic nanomaterials could be considered for STO implementation, including high- $\alpha$ materials, provided that the spin polarization of the electron flow is retained. 


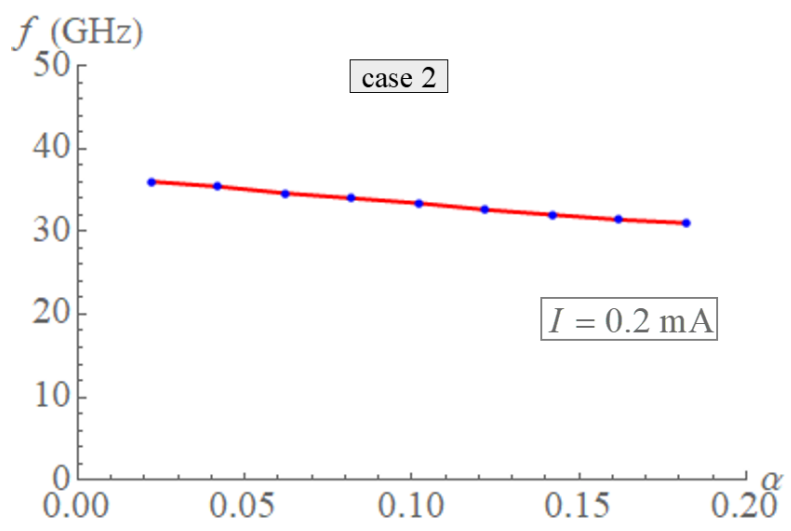

Figure S12: Frequency of precession as a function of free-layer damping constant for a given applied electric current in case 2.

Figure S13 shows that the oscillation frequency is, in case 2, inversely proportional to the square of the free-layer thickness, similarly as in case 0 and case 1. Effective variations in free-layer length caused by local defects in fabricated devices are, consequently, expected to broaden more significantly spectral linewidths of STOs having shorter free layers in the three cases considered.

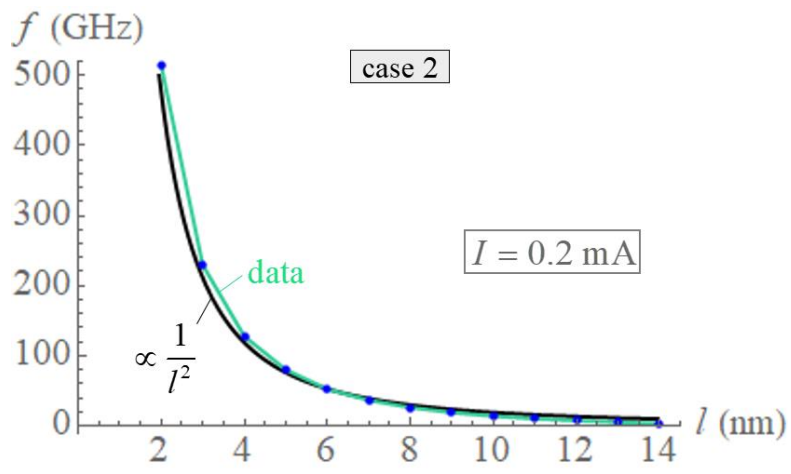

Figure S13: Frequency of precession as a function of free-layer length for a given applied electric current in case 2 , showing an inverse square dependence.

Figure S14a shows how the free-layer perpendicular-magnetocrystalline-anisotropy energy density influences the frequency-current dependence. Since in case 2, for the considered STO specification, all spins have a positive or near-zero vertical component (Fig. 4c(ii)), it means that the perpendicularmagnetocrystalline-anisotropy field, given by Eq. (A3), has the same upward direction for each spin. Consequently, the resulting perpendicular-magnetocrystalline-anisotropy torque assists in rotating the spin chain in the CCW direction. As Fig. S14a illustrates, increasing $K_{\mathrm{u}}$ results in an expansion of the range of 
currents over which CCW precession $(f>0$ ) occurs. Figure S14b shows the frequency dependence on $K_{\mathrm{u}}$ for two different current values.
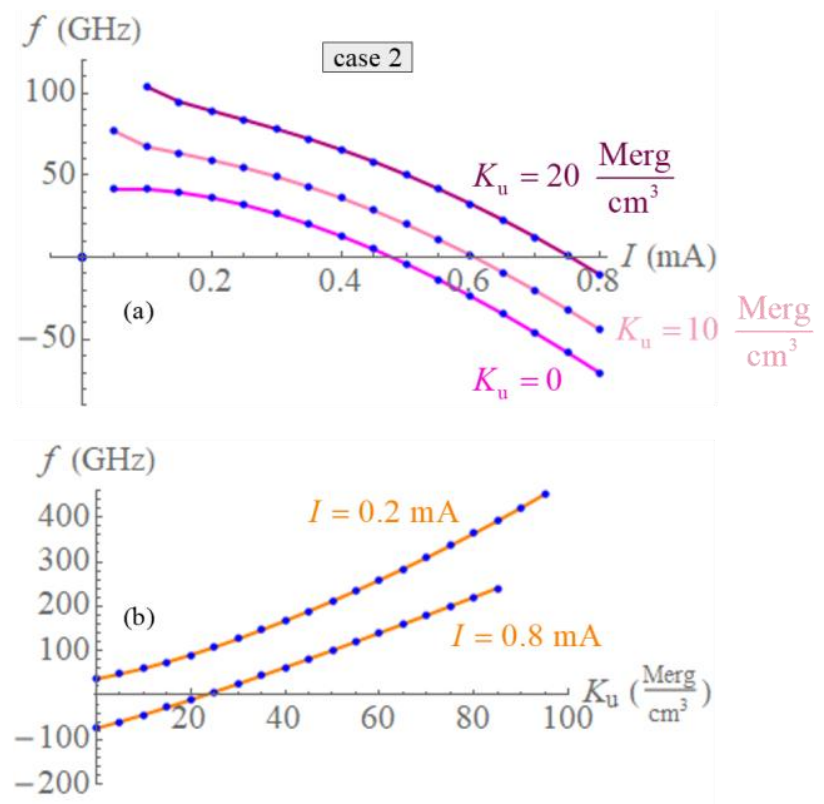

Figure S14: (a) Frequency-current characteristics for several illustrative values of perpendicularmagnetocrystalline-anisotropy energy density $K_{u}$ of the free layer in case 2. (b) Precessional frequency as a function of $K_{\mathrm{u}}$ for two different applied current amplitudes.

By modulating the strength of the spin polarization $P$ of the applied electric current within the free magnetic layer through materials selection and fabrication techniques, the frequency-current characteristics can be modified. Figure S15a shows the $f-I$ characteristics for three values of the spin polarization. For $P=0$ the ${ }^{f-I}$ characteristic obtained is equivalent to that of case 0 (Fig. 2a). As we increase $P$, the bulk STT tends to increasingly translate the DW toward the polarizer layer, which prompts a competition between the azimuthal components of the bulk STT and the interfacial STT, which for a given value of applied current results in a static equilibrium of the spin chain, i.e., no precession ( $f=0$ ). The greater the spin polarization $P$ within the free layer, the lower is the current for which compensation is achieved. Figure S15b illustrates how the frequency of precession varies with the spin polarization for two different current values. The relative modulation of the strengths of the bulk and interfacial STTs through modulation of the spin polarization, or equivalently, through the modulation of the magnetoresistance associated with the polarizer/spacer/free-layer stack, represents an additional means of optimization of STO characteristics. 

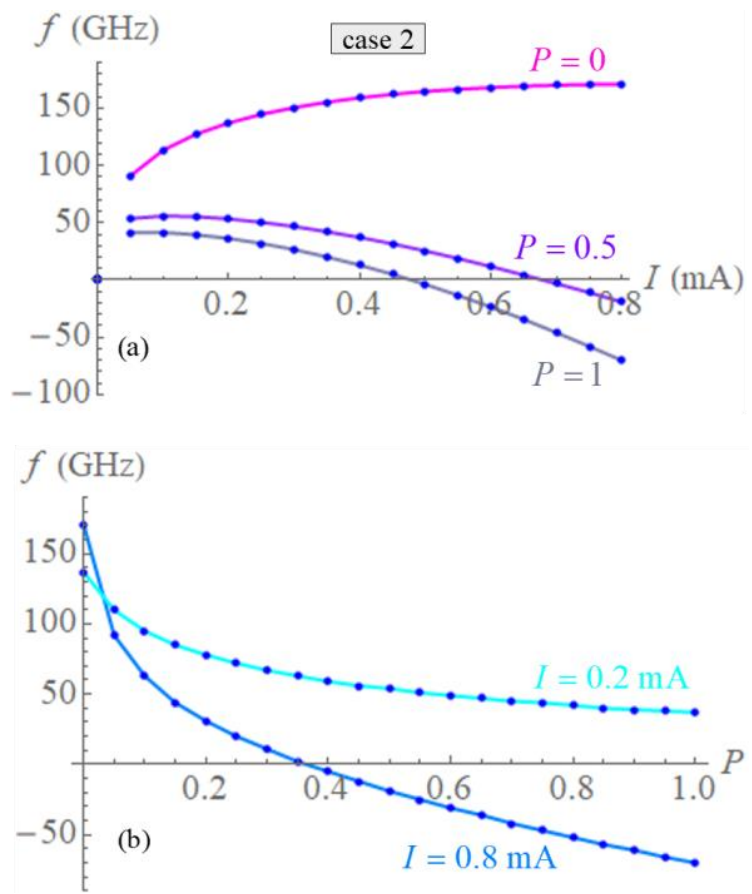

Figure S15: (a) Frequency-current characteristics for several illustrative values of spin polarization $P$ of electric current within the free layer in case 2. (b) Precessional frequency as a function of $P$ for two different applied current amplitudes. 